\title{
A CENTRAL LIMIT THEOREM FOR RANDOM WALKS IN RANDOM LABYRINTHS
}

\author{
Carol Bezuidenhout and Geoffrey Grimmett
}

\begin{abstract}
A beam of light shines through the lattice $\mathbb{Z}^{d}$, and is subjected to reflections determined by a random environment of mirrors at the vertices of $\mathbb{Z}^{d}$. The behaviour of the light ray is investigated under the hypothesis that the environment contains a strictly positive density of vertices at which the light behaves in the manner of a random walk. When $d \geq 2$ and the density of non-trivial reflectors is sufficiently small, the environment contains a.s. a unique infinite 'inter-illuminating' class of vertices. Furthermore, when the light beam originates within this class, then its trajectory obeys a functional central limit theorem with a strictly positive diffusion constant. These facts are obtained using percolation-type arguments, together with the invariance principle proposed by Kipnis and Varadhan.
\end{abstract}

\section{Introduction}

What is the behaviour of a beam of light passing through a medium of reflecting bodies? Such an investigation was initiated by Lorentz [26] nearly a century ago in a formulation which has come to be known as the 'Lorentz gas'. Suitably reformulated for the latter-day mathematician, one version of the question becomes the following. Suppose that smooth bodies are distributed randomly about $d$-dimensional space $\mathbb{R}^{d}$ according to some given probability measure. A ray of light originates from a specified point of $\mathbb{R}^{d}$, travelling initially in a specified direction; this ray passes through $\mathbb{R}^{d}$ subject to reflections at the surfaces of these bodies. We now ask for properties of the trajectory. For example, what can be said about the displacement of the point which is distance $t$ along the path, in the limit of large $t$ ?

The work of Lorentz inspires a lattice model in which, conditional on the environment of reflecting bodies, the trajectory of the light is deterministic. Remarkably little is known about such systems in general, although some progress has been made in the special case of the square lattice towards deciding whether or not the light beam is confined to a bounded region of space; see [5, 17, 18, 28].

Several authors have considered stochastic relaxations of such lattice systems. In one possible such relaxation, one allows random deviations from the rules whenever light impacts on a mirror. Subject to certain conditions including one of irreducibility, the light ray then (a.s.) illuminates the whole space and satisfies a central limit theorem; see [29].

We pursue another route here, namely that discussed in $[2,3,21,22]$ and involving the introduction of a positive density of points (or 'scatterers') at which the light beam behaves in the manner of a random walk. This leads to a model which is partially tractable using probabilistic analysis, but which poses substantial difficulties arising from the geometrical constraints of the environment of mirrors. We show in this paper how the geometry of the environment may be controlled using 'block' arguments taken from percolation theory. In

1991 Mathematics Subject Classification. 60J15, 60K35, 60F15, 82C41.

Key words and phrases. Random walk, random labyrinth, central limit theorem, invariance principle.

This version was prepared on 26 February 1998 
this way, we extend the non-localisation theorem of [21] in order to obtain a central limit theorem. This last theorem may be viewed as a generalisation of certain results in [11, 22].

Next we present an illustration of such a stochastic relaxation associated with the twodimensional square lattice $\mathbb{Z}^{2}$. Let $p_{\text {rw }}, p_{\text {nw }}, p_{\text {ne }}$ be non-negative numbers whose sum satisfies $p_{\text {rw }}+p_{\text {nw }}+p_{\text {ne }} \leq 1$. Each vertex of $\mathbb{Z}^{2}$ is allocated a random state from the local state space $\{\mathrm{rw}, \mathrm{nw}, \mathrm{ne},+\}$ where the probability of state $\sigma$ is $p_{\sigma}$, and where $p_{+}=1-p_{\mathrm{rw}}-p_{\mathrm{nw}}-$ $p_{\text {ne }}$; different vertices are allocated independent states. These states are interpreted in the following way.

(a) A vertex labelled 'rw' is called a 'random walk (rw) point'. Light incident with a rw point behaves as a symmetric random walk, in the sense that it departs the vertex in a direction chosen randomly from the set of four possible directions, this choice being made independently of all vertex states and of all previous choices.

(b) A vertex labelled 'nw' is occupied by a NW mirror, which is to say that northerly light is reflected westwards, westerly light is reflected northwards, southerly light is reflected eastwards, easterly light is reflected southwards.

(c) A vertex labelled 'ne' is occupied by a NE mirror, which is defined similarly but with north and south interchanged.

(d) A vertex labelled ' + ' is called a 'crossing'. Light incident with a crossing passes directly through without deviation.

We now shine light from the origin in a specified initial direction, and first ask the obvious question of whether or not the set of illuminated vertices is a.s. finite. The problem of main interest for ergodic theorists is the case when $p_{\mathrm{rw}}=0$, for which, conditional on the environment, the light behaves deterministically. Under the contrasting assumption that $p_{\text {rw }}>0$, it has been shown in [21] that the illuminated set is infinite with strictly positive probability if $p_{\mathrm{nw}}+p_{\mathrm{ne}}$ is sufficiently small (and positive). In contrast to certain other arguments which are specific to this two-dimensional system, the conclusion of "nonlocalisation' was obtained in [21] for general systems of reflectors in $\mathbb{Z}^{d}$ where $d \geq 2$, whenever the density $p_{\text {rw }}$ of random walk points is strictly positive, and the density of non-trivial reflectors (i.e., reflectors other than crossings) is sufficiently small.

Assume now that the number $d$ of dimensions satisfies $d \geq 2$. Let us consider a situation in which $p_{\mathrm{rw}}>0$, and where light originating at the origin illuminates infinitely many vertices. Let $Y(n)$ be the (random) displacement of the light after it has travelled a total distance $n$ (i.e., it has traversed exactly $n$ edges). Our purpose in this paper is to prove a central limit theorem for $Y(n)$. We shall prove, subject to suitable conditions, that the $d$ coordinates of $Y(n)$ are (asymptotically) independent normal random variables with zero mean and variance $\delta n$, where the diffusion constant $\delta$ depends on the parameters of the system and is strictly positive.

We may think of the 'random field' of reflectors as a special type of random environment having a great deal of rigidity. Our basic strategy in proving the central limit theorem is to adapt the arguments proposed by Kipnis and Varadhan [24] and further developed by DeMasi, Ferrari, Goldstein, and Wick [10, 11]. Substantial difficulties arise in following this strategy. Whereas the above papers considered reversible random walks in a random environment on $\mathbb{Z}^{d}$, we shall need here to study the Markov chain embedded in the light path by looking only at those times at which the light passes through rw points. The set of rw points is a random set, and the geometry of this set will be controlled using percolationtheoretic arguments. Indeed, the majority of this paper is devoted to obtaining and applying estimates for the geometry of the environment. The technology for proving the central limit 
theorem is itself taken off the peg from [11, 24]; the main problems of the current paper are to prove that the methods of $[11,24]$ are applicable in the current setting, and to verify that the resulting diffusion constant is strictly positive.

It is a matter of substantial interest whether or not a light trajectory is 'diffusive' when the density $p_{\mathrm{rw}}$ of rw points equals 0. Extensive Monte Carlo simulations have been carried out $([7,8,9,30,31])$, but little of mathematical rigour is currently known concerning this hard question.

The requisite definitions are given in the next section, and the central limit theorem is stated at the end of that section. In Section 3, we present certain lemmas concerning the geometry of the set of points illuminated by light originating at the origin. Section 4 contains several estimates concerning the conductance of a certain disordered electrical network derived from a random labyrinth. We prove the central limit theorem in Section 5 , but reserve until Section 6 the proof that the diffusion constant is strictly positive. This last step is achieved, as in [11], by utilising the electrical results of Section 4 .

\section{Random labyrinths}

Random labyrinths were introduced in $[2,3,21,22]$ and discussed further in [18]. We describe here a general labyrinthine model for the passage of light through the cubic lattice in $d$ dimensions, where $d \geq 2$. By $\mathbb{Z}^{d}$, we mean the set of all $d$-vectors $v=\left(v_{1}, v_{2}, \ldots, v_{d}\right)$ of integers. We shall use the norms $|x|=\sum_{i=1}^{d}\left|x_{i}\right|$ and $\|x\|=\sup \left\{\left|x_{i}\right|: 1 \leq i \leq d\right\}$ for $x=\left(x_{1}, x_{2}, \ldots, x_{d}\right) \in \mathbb{Z}^{d}$. The set $\mathbb{Z}^{d}$ is turned into a graph by adding edges $\langle x, y\rangle$ between all pairs $x, y \in \mathbb{Z}^{d}$ satisfying $|x-y|=1$. The ensuing graph is denoted $\mathbb{L}^{d}=\left(\mathbb{Z}^{d}, \mathbb{E}^{d}\right)$ and the origin is written as 0 .

Let $I=\left\{u_{1}, u_{2}, \ldots, u_{d}\right\}$ where $u_{i}=(0, \ldots, 0,1,0, \ldots, 0)$ is the unit vector in the $i$ th coordinate direction, and let $I^{ \pm}=\{-1,+1\} \times I$ be the set of all $\pm u_{i}$. We define a reflector to be a map $\rho: I^{ \pm} \rightarrow I^{ \pm}$with the property that $\rho(-\rho(u))=-u$ for all $u \in I^{ \pm}$, and we write $\mathcal{R}$ for the set of all reflectors.

The physical interpretation of a reflector $\rho$ is as follows. If light is incident at a vertex $x$ in direction $u\left(\in I^{ \pm}\right)$, the effect of reflector $\rho$ at $x$ is to deflect the light ray in such a way that it departs $x$ in direction $\rho(u)$. The condition $\rho(-\rho(u))=-u$ is in response to the reversibility of light paths, and it plays a role in the probabilistic arguments which follow.

We distinguish two special reflectors, as follows. The identity mapping on $I^{ \pm}$is called the crossing, denoted by + . Crossings do not deflect light beams. The reflector $\rho$ satisfying $\rho(u)=-u$ (for all $u$ ) is called the blocker, and is denoted by $\square$. It has the effect of reflecting any ray of light back upon itself.

Let $\mu$ be a probability measure on the set $\mathcal{R} \cup\{\varnothing\}$. We place a member of $\mathcal{R} \cup\{\varnothing\}$ at each vertex $x$, this member being sampled according to $\mu$, and in such a way that different vertices are occupied by independent members. That is, we consider the environment space

$$
\Omega=\{\mathcal{R} \cup\{\varnothing\}\}^{\mathbb{Z}^{d}},
$$

and let $\mathbb{P}$ be product measure on $\Omega$ with marginals $\mu$. We introduce two parameters which will play important roles later, namely

$$
p_{\text {rw }}=\mu(\{\varnothing\}), \quad p_{+}=\mu(\{+\}),
$$

the densities of the local states $\varnothing$ and + (recall that + denotes the crossing). The notation will become clear soon. 


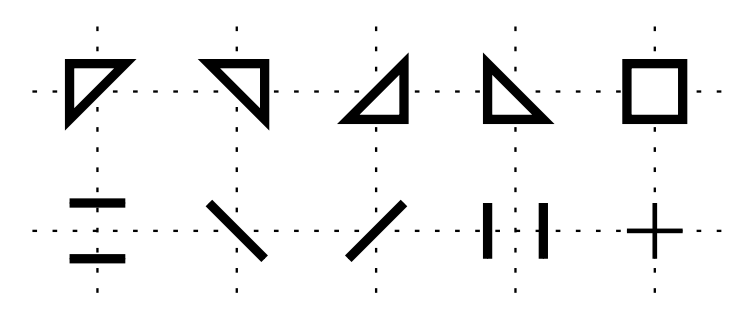

Fig. 1. There are 10 possible reflectors in two dimensions. The blocker is $\square$, the crossing + , and the other icons represent the more complicated reflectors.

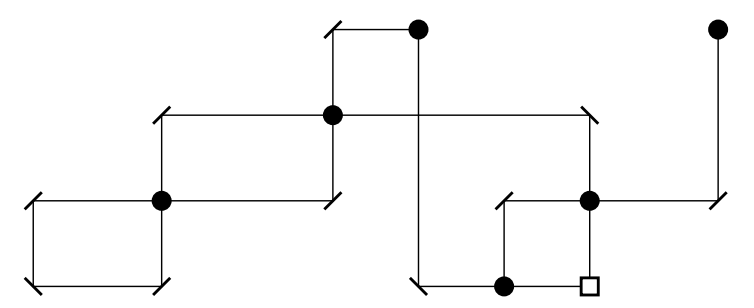

Fig. 2. A sketch of some two-dimensional light paths joining rw points (represented by $\bullet$ ). Note the existence of loops, parallel paths, crossing paths, and blocked paths.

Given an environment $\omega(\in \Omega)$, we wish to construct a random walk in $\omega$. Such a walk will conform to the reflectors, but will behave in the manner of a symmetric random walk whenever it arrives at a point in state $\varnothing$. See Figures 1 and 2 for illustrations of random walks through two-dimensional labyrinths.

For $\omega=\left(\omega_{x}: x \in \mathbb{Z}^{d}\right) \in \Omega$, let $\mathcal{W}(\omega)=\left\{x \in \mathbb{Z}^{d}: \omega_{x}=\varnothing\right\}$, and call $\mathcal{W}(\omega)$ the set of random walk (or rw) points in the configuration $\omega$. A path in $\mathbb{L}^{d}$ is an ordered sequence $v_{0}, v_{1}, \ldots, v_{n}$ of vertices (not necessarily distinct) such that $\left\langle v_{k}, v_{k+1}\right\rangle \in \mathbb{E}^{d}$ for $0 \leq k<n$. A light path in $\omega$ is a path $v_{0}, v_{1}, \ldots, v_{n}$ with $n \geq 1$ such that

(a) $v_{k}$ is a rw point if and only if $k \in\{0, n\}$,

(b) for $k \geq 2$, we have that $v_{k}-v_{k-1}=\omega_{v_{k-1}}\left(v_{k-1}-v_{k-2}\right)$.

Informally, a light path is the trajectory of light which departs the rw point $v_{0}$ in the direction $v_{1}-v_{0}$, up to the moment when the light illuminates a rw point for the next time. Such a path is said to connect its endpoints.

For rw points $x, y$, let $n^{\omega}(x, y)$ be the number of light paths connecting $x$ to $y$. For sets $A, B$ of rw points, we set

$$
n^{\omega}(A, B)=\sum_{\substack{x \in A \\ y \in B}} n^{\omega}(x, y) ;
$$

$n^{\omega}(x, B)$ and $n^{\omega}(A, y)$ are defined accordingly. We define an equivalence relation ' $\leftrightarrow$ ' on $\mathcal{W}(\omega)$ by $x \leftrightarrow y$ if either $x=y$, or there is a sequence $x_{0}=x, x_{1}, \ldots, x_{m}=y$ of rw points, where $m \geq 1$, such that

$$
\prod_{k=1}^{m} n^{\omega}\left(x_{k-1}, x_{k}\right)>0 .
$$

Any rw point $x$ lies in some equivalence class $C^{\omega}(x)$ of the relation $\leftrightarrow$. We shall usually write $n$ and $C$ for $n^{\omega}$ and $C^{\omega}$, except where such notation would be ambiguous for the context.

Let $\omega \in \Omega$. We define a random walk in the labyrinth $\omega$ to be a Markov chain, denoted 
$\left(X^{\omega}(n): n \geq 0\right)$, having state space $\mathcal{W}(\omega)$ and transition matrix $q^{\omega}$ given by

$$
q^{\omega}(x, y)=\frac{n^{\omega}(x, y)}{2 d} \quad \text { for } x, y \in \mathcal{W}(\omega)
$$

Such a random walk $X^{\omega}$ is the main object of study of this paper. We denote by $P_{x}^{\omega}$ the law of $X^{\omega}$ conditional on $X^{\omega}(0)=x$.

One of the main properties of the chain $X^{\omega}$ is its reversibility (relative to an appropriate measure), and it is this that permits the use of the arguments of $[11,24]$. If we were to permit rules more general than those given above, then this vital property would generally no longer hold.

It is clear from the definition of the equivalence relation $\leftrightarrow$ that the communicating classes of $X^{\omega}$ are exactly the equivalence classes of $\leftrightarrow$. The asymptotic behaviour of $X^{\omega}(n)$ for large $n$ is an interesting object of study only if the starting point $X^{\omega}(0)$ lies in an infinite equivalence class of $\leftrightarrow$. We therefore introduce the subspaces of $\Omega$ given by

$$
\Omega^{*}=\left\{\omega \in \Omega: \omega_{0}=\varnothing\right\}, \quad \Omega^{* *}=\left\{\omega \in \Omega^{*}:\left|C^{\omega}(0)\right|=\infty\right\},
$$

and we write $\mathbb{P}^{*}$ and $\mathbb{P}^{* *}$ for the measure $\mathbb{P}$ conditioned respectively on the events $\Omega^{*}$ and $\Omega^{* *}$. (These measures are defined whenever $\mathbb{P}\left(\Omega^{*}\right)>0$ and $\mathbb{P}\left(\Omega^{* *}\right)>0$.)

We assume that $X^{\omega}(0)=0$, and define the re-scaled variables

$$
X^{\varepsilon, \omega}(t)=\varepsilon X^{\omega}\left(\left\lfloor\varepsilon^{-2} t\right\rfloor\right), \quad \varepsilon>0, t>0 .
$$

By the term 'standard Brownian motion' we mean a Wiener process with the identity covariance matrix. If $W$ is a standard Brownian motion in $\mathbb{R}^{d}$ and $\mathbf{C}$ is a real $d \times d$ matrix, then $\mathbf{C W}$ is a Wiener process with covariance matrix $\mathbf{C C}^{\prime}$.

Throughout this paper, we write $p_{\mathrm{c}}=p_{\mathrm{c}}\left(\mathbb{L}^{d}\right.$, site) for the critical probability of site percolation on $\mathbb{L}^{d}$. The following theorem utilises a type of convergence denoted in the form ' $\mathbb{P}^{* *}$-dp'; the appropriate definition appears after the statement of the theorem.

Theorem 2.1. Let $p_{\mathrm{rw}}>0$. There exists a strictly positive constant $A=A\left(p_{\mathrm{rw}}\right)$ such that the following holds whenever either $1-p_{\mathrm{rw}}-p_{+}<A$ or $p_{\mathrm{rw}}>p_{\mathrm{c}}$ :

(a) $\mathbb{P}\left(\Omega^{* *}\right)>0$,

(b) as $\varepsilon \downarrow 0$, the re-scaled process $X^{\varepsilon,}$, converges $\mathbb{P}^{* *}$-dp to $\sqrt{D} W$, where $W$ is a standard Brownian motion in $\mathbb{R}^{d}$ and $D=D(\mu)$ is a strictly positive constant.

We recall that $\mu$ is the marginal measure of $\mathbb{P}$. In saying that $X^{\varepsilon, \cdot}$ converges $\mathbb{P}^{* *}$-dp to $\sqrt{D} W$, we mean that, as $\varepsilon \downarrow 0$,

$$
P_{0}^{\omega}\left(f\left(X^{\varepsilon, \omega}\right)\right) \rightarrow E(f(\sqrt{D} W)) \quad \text { in } \mathbb{P}^{* *} \text {-probability }
$$

for all bounded continuous functions $f$ on the Skorohod space $D\left([0, \infty), \mathbb{R}^{d}\right)$. [For any random variable $Z$ and appropriate probability measure $P$, we write $P(Z)$ for the mean of $Z$. Here, $E$ is the expectation operator for the Brownian motion $W$.] In this two tiered mode of convergence, the letters 'dp' stand for 'in distribution in probability'.

Theorem 2.1 asserts a functional central limit theorem for the random walk $X^{\omega}$ when it is confined to an infinite equivalence class, under the assumption that the density of nontrivial reflectors (i.e., reflectors other than the crossing) is sufficiently small. Note that $X^{\omega}$ does not conform to 'real time'; that is, it jumps between rw points at unit times, rather than following the light trajectory at constant velocity. Actually it may be viewed as the 
embedded chain obtained by observing a 'real time' process at the epochs of visits to rw points. There is a central limit theorem for such a 'real time' process also.

Let $\omega \in \Omega$. We define a Markov chain $\left(Z^{\omega}(n): n \geq 0\right)$ on the state space $I^{ \pm} \times \mathbb{Z}^{d}$ as follows. Writing $Z^{\omega}(n)=\left(U^{\omega}(n), Y^{\omega}(n)\right) \in I^{ \pm} \times \mathbb{Z}^{d}$, we require that

$$
\begin{aligned}
& Y^{\omega}(n+1)=Y^{\omega}(n)+U^{\omega}(n+1), \\
& U^{\omega}(n+1)=\omega_{Y^{\omega}(n)}\left(U^{\omega}(n)\right) \text { if } Y^{\omega}(n) \text { is not a rw point; }
\end{aligned}
$$

if $Y^{\omega}(n)$ is a rw point, then $U^{\omega}(n+1)$ is chosen uniformly from $I^{ \pm}$, this choice being independent of $\omega$ and of all earlier choices. As before, we set $Y^{\varepsilon, \omega}(t)=\varepsilon^{-1} Y^{\omega}\left(\left\lfloor\varepsilon^{-2} t\right\rfloor\right)$.

Theorem 2.2. Let $p_{\mathrm{rw}}>0$ and let $A=A\left(p_{\mathrm{rw}}\right)$ be given as in Theorem 2.1. If either $1-p_{\mathrm{rw}}-p_{+}<A$ or $p_{\mathrm{rw}}>p_{\mathrm{c}}$, then the re-scaled process $Y^{\varepsilon, \cdot}$ converges $\mathbb{P}^{* *}$-dp to $\sqrt{\delta} W$, where $W$ is a standard Brownian motion in $\mathbb{R}^{d}$ and $\delta=\delta(\mu)$ is a strictly positive constant.

The two diffusion constants $D$ and $\delta$ are related in the following way. Suppose 0 is a rw point, and let $x_{1}, x_{2}, \ldots, x_{2 d}$ be the vertices $x$ of $\mathbb{Z}^{d}$ (appearing with the appropriate multiplicities) with the property that $n^{\omega}(0, x)>0$. Let $l_{1}, l_{2}, \ldots, l_{2 d}$ be the numbers of edges in the corresponding light paths. Then $\delta=D / m$ where

$$
m=\mathbb{P}^{* *}\left(\frac{1}{2 d} \sum_{k=1}^{2 d} l_{k}\right) .
$$

The proof of this theorem may be found in Section 7 .

Our principal theorem, Theorem 2.1, concerns the discrete-time process $X^{\omega}(n)$. A similar conclusion is valid for a process in continuous time. At one point in the proof, we shall need to refer to such a process, and therefore we introduce it here. Specifically, we let $\left(X_{t}^{\omega}: t \geq 0\right)$ be the 'Poissonization' of $X^{\omega}$ given in the usual (following) way. Let $(M(t): t \geq 0$ ) be a Poisson process with rate 1 , independent of all random variables so far considered, and let $X_{t}^{\omega}=X^{\omega}(M(t))$. Then $X_{t}^{\omega}$ is a Markov process which follows the trajectories of $X^{\omega}(\cdot)$ but with exponentially-distributed holding times at each rw point.

Finally in this section, we consider the special case when $p_{\mathrm{rw}}>p_{\mathrm{c}}$ and when all non-rw points are (a.s.) blockers. In this case, the light moves in the manner of a random walk between the rw points, and it experiences a delay whenever it exits a rw point in the direction of a blocker. This process constitutes essentially a random walk on the infinite cluster of a supercritical site percolation process, with holding times at each rw point $x$ having a distribution depending on the number of neighbouring blockers at $x$. Such a process was considered in [11], and the results of the present paper contain a certain generalisation of the corresponding central limit theorem presented there.

\section{Geometrical properties of labyrinths}

In order to establish results concerning a light path in a labyrinth, it is first necessary to derive some geometrical properties of the labyrinth. Several basic properties will be required, and we present these next.

Let $\omega \in \Omega$, and consider the equivalence relation $\leftrightarrow$ on the set $\mathcal{W}(\omega)$ of rw points. Let $M=M(\omega)$ be the number of infinite equivalence classes. The following uniqueness theorem will be proved later in this section using the approach of Burton and Keane [6], with variations. 
Theorem 3.1. Suppose $p_{\mathrm{rw}}>0$. Then either $\mathbb{P}(M=0)=1$ or $\mathbb{P}(M=1)=1$.

It was proved in [21] that light paths are usually rather short in length. Let $e=\langle u, v\rangle$ be an edge. Either $e$ lies in some light path $v_{0}, v_{1}, \ldots, v_{k}$ where $v_{0}$ and $v_{k}$ are rw points (and where $v_{0}=v_{k}$ is allowed), or it does not. We write $\lambda(e)=k$ if this path exists having length $k$, and $\lambda(e)=0$ otherwise. It is clear that $\lambda(e)$ is well defined.

Theorem 3.2. For any edge e,

$$
\mathbb{P}(\lambda(e)>2 k) \leq 2\left(1-p_{\text {rw }}\right)^{(k-1) /(2 d)} \quad \text { for } k \geq 1 .
$$

The proof may be found in $[18,21]$, but for the sake of being complete we summarise it here. Light passing along $e$ from $u$ to $v$ will continue through $\mathbb{Z}^{d}$ until it meets a rw point for the first time. At each new point that it encounters, this point is a rw point with probability $p_{\mathrm{rw}}$, and the chance that $r$ new points are not rw points is therefore $\left(1-p_{\mathrm{rw}}\right)^{r}$. The coefficient 2 arises because $e$ may be traversed in either of two directions; the factor $(2 d)^{-1}$ in the exponent arises since no vertex is visited more than $2 d$ times by any given light path.

We turn now to the sizes of the finite equivalence classes of $(\mathcal{W}(\omega), \leftrightarrow)$. We augment the equivalence class $C(x)$ at each rw point $x$ by adding all vertices of $\mathbb{Z}^{d}$ on all light paths connecting vertices in $C(x)$, and we denote the augmented set of vertices by $\bar{C}(x)$. The radius of a set $A$ of vertices containing the origin is given by

$$
\operatorname{rad}(A)=\max \{|x|: x \in A\}
$$

and its boundary $\partial A$ is the set of vertices $x(\in A)$ which are adjacent to some vertex $y$ not in $A$. Recall that $\mathbb{P}^{*}$ denotes $\mathbb{P}$ conditioned on the event $\Omega^{*}$ that the origin is a rw point.

Theorem 3.3. Suppose that $p_{\mathrm{rw}}>0$. There exist strictly positive constants $A=A\left(p_{\mathrm{rw}}\right)$, $\psi=\psi\left(p_{\text {rw }}\right)$, such that $\mathbb{P}^{*}(|C(0)|=\infty)>0$ and

$$
\mathbb{P}^{*}(k \leq \operatorname{rad}(\bar{C}(0))<\infty)<e^{-k \psi} \quad \text { for } k \geq 1
$$

whenever either $1-p_{\mathrm{rw}}-p_{+}<A$ or $p_{\mathrm{rw}}>p_{\mathrm{c}}$.

Further geometrical properties of this type may be established, using comparisons with a percolation process, but we shall use only the above. In proving Theorem 3.3 we shall make use of a 'block argument' which has other applications too. This argument is similar to one presented in [21].

Consider the box $B^{N}=[-N, N-1]^{d}$. We shall introduce a property of $B^{N}$ which will depend only on $\omega$ restricted to $B^{N}$. Roughly speaking, this property is that: $B^{N}$ contains no reflectors other than crossings, and light originating anywhere in $B^{N}$ will illuminate the whole of $B^{N}$. In order to achieve a proper definition, we introduce the following terminology.

For $\omega \in \Omega$, we use the term $\omega$-path to mean a path $v_{0}, v_{1}, \ldots$ of $\mathbb{Z}^{d}$ with the property that, for all $j \geq 1$,

$$
v_{j+1}-v_{j}=\omega_{v_{j}}\left(v_{j}-v_{j-1}\right) \text { whenever } v_{j} \text { is not a rw point, }
$$

which is to say that the path conforms to the reflectors at all non-rw points. For $x, y \in \mathbb{Z}^{d}$, we write $x \leadsto y$ if there exists an $\omega$-path with endpoints $x$ and $y$. For any box $T$, we write $x{ }^{\prime}{ }_{T} y$ if there exists an $\omega$-path with endpoints $x$ and $y$ which has at most one vertex lying outside $T \backslash \partial T$. If $T=B^{N}$, we write $\varkappa_{N}$ for $\varkappa_{T}$. 
We declare the box $B^{N}$ to be good if the two following properties hold:

(a) $B^{N}$ contains only crossings and rw points,

(b) there exists a rw point $x$ in $B^{N / 2}$ such that $x \varkappa_{N} y$ for all $y \in \partial B^{N}$.

[We call any such point $x$ a seed of the good box $B^{N}$.] Similarly, we call a translate $T=v+B^{N}$ good if it contains only crossings and rw points, and there exists a rw point $x \in v+B^{N / 2}$ such that $x{ }^{\prime}{ }^{\prime} y$ for all $y \in \partial T=v+\partial B^{N}$; such a point $x$ is called a seed of the translate.

Theorem 3.4. Suppose that $p_{\mathrm{rw}}>0$ and $\eta>0$. There exists a strictly positive constant $A=A\left(p_{\mathrm{rw}}, \eta\right)$ and a positive integer $M$ such that

$$
\mathbb{P}\left(B^{N} \text { is good }\right)>1-\eta \quad \text { for } N \geq M
$$

whenever $1-p_{\text {rw }}-p_{+}<A$.

The value of this theorem is as follows. For $l \in \mathbb{Z}^{d}$, we colour $l$ green if the translate $B_{l}^{N}=2 N l+B^{N}$ is good. By choosing $\eta$ small enough, we can make the density of green sites close to 1 , and in particular bigger than the critical probability of site percolation on $\mathbb{Z}^{d}$. Furthermore, the definition of 'good' entails that any cluster of green sites corresponds to a collection of good boxes in $\mathbb{Z}^{d}$ whose rw points lie in the same equivalence class of $(\mathcal{W}(\omega), \leftrightarrow)$. By using percolation estimates for such clusters, we obtain information about the geometry of equivalence classes. In particular, if there exists an infinite cluster of green sites on the renormalised lattice, then the corresponding region of the labyrinth contains an infinite 'inter-illuminating' class of rw points.

Proof of Theorem 3.1. Since $M$ is a translation-invariant function on $\Omega$, and since $\mathbb{P}$ is ergodic, we deduce that there exists a constant $m \in\{0,1,2, \ldots\} \cup\{\infty\}$ such that $\mathbb{P}(M=$ $m)=1$. We can rule out the possibility that $2 \leq m<\infty$ as follows. If $2 \leq m<\infty$, then there exists an integer $N$ such that

$$
\mathbb{P}\left(B^{N} \text { intersects } m \text { infinite equivalence classes }\right)>\frac{1}{2} .
$$

We may place a rw point at every vertex in $B^{N}$, thereby causing the $m$ infinite equivalence classes to coalesce into a single such class. It would follow that $\mathbb{P}(M=1)>0$, a contradiction.

It remains to rule out the case $m=\infty$, and to this end we assume henceforth that $\mathbb{P}(M \geq 3)=1$. For any $\omega \in \Omega$ and any finite box $B$, we denote by $\omega_{B}^{\square}$ the following configuration:

$$
\omega_{B}^{\square}(x)= \begin{cases}\square & \text { if } x \in \partial B, \\ \omega(x) & \text { otherwise }\end{cases}
$$

where $\square$ denotes the blocker. Let $N$ be a positive integer, and call a translate $E=v+B^{N}$ of the box $B^{N}$ an encounter zone if the following holds:

(a) $E$ contains only rw points,

(b) the unique equivalence class of $(\mathcal{W}(\omega), \leftrightarrow)$ containing points in $E$ is infinite, and may be partitioned as $C_{1} \cup C_{2} \cup \ldots \cup C_{r} \cup F \cup E$, where $r=r(E) \geq 3$, and where $C_{1}, C_{2}, \ldots, C_{r}$ are distinct infinite equivalence classes and $F$ is a union of finite equivalence classes of $\left(\mathcal{W}\left(\omega_{E}^{\square}\right), \leftrightarrow\right)$.

Loosely speaking, an encounter zone $E$ has the property that it 'welds together' three or more infinite equivalence classes of $\mathbb{Z}^{d} \backslash E$. Note that encounter zones may overlap one another. 


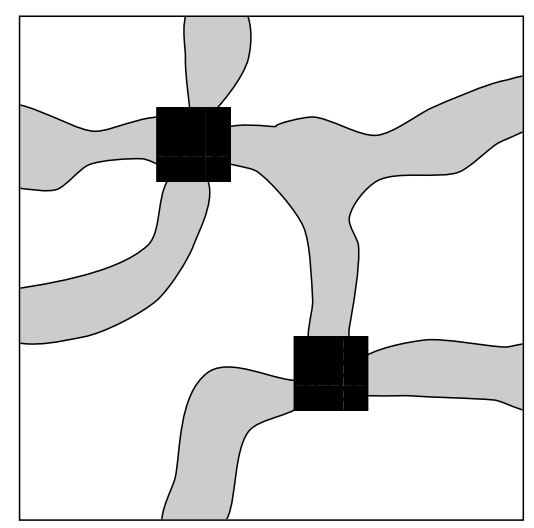

Fig. 3. Two encounter zones, generating compatible partitions.

Some care is needed in order to follow the strategy laid down by Burton and Keane [6]. First, it follows by the construction given above that $\mathbb{P}\left(B^{N}\right.$ is an encounter zone $)=\eta>0$ for some $N$, and we pick $N$ accordingly. It follows by translation-invariance that

$$
\mathbb{P}(E \text { is an encounter zone })=\eta>0
$$

for all translates $E=v+B^{N}$ of $B^{N}$.

Next, write $\Delta G$ for the set of edges of the lattice having exactly one endpoint in the set $G$ of vertices. We shall consider the set of encounter zones contained within a large box $B^{n}$. Let $E=v+B^{N}$ be such an encounter zone, and let $\Delta_{n} E$ be the set of edges $e$ in $\Delta B^{n}$ with the following properties:

(i) $e$ belongs to some $\omega_{E}^{\square}$-path which visits infinitely many distinct rw points, and

(ii) $e$ lies in some $\omega$-path $\pi$ using edges of $B^{n}$ only (apart from $e$ itself), such that $\pi$ has an endpoint in $E$.

Using property (b) of the definition of encounter zone, we have that $\Delta_{n} E$ may be partitioned into the union of non-empty sets $\Delta_{n} E^{1}, \ldots, \Delta_{n} E^{r}$ where $r=r(E) \geq 3$, and with the following property: for every $j$, there exists an infinite equivalence class of $\left(\mathcal{W}\left(\omega_{E}^{\square}\right), \leftrightarrow\right)$ such that all edges in $\Delta_{n} E^{j}$ lie in $\omega$-paths joining rw points of this class.

Now let $E_{1}$ and $E_{2}$ be distinct encounter zones contained within $B^{n}$. (Note that $E_{1}$ and $E_{2}$ may have non-empty intersection.) It may be seen (aided perhaps by Figure 3) that: either $\Delta_{n} E_{1} \cap \Delta_{n} E_{2}=\varnothing$, or the partitions corresponding to $\Delta_{n} E_{1}$ and $\Delta_{n} E_{2}$ are 'compatible' in the sense that there exist orderings of the sequences $\Delta_{n} E_{1}^{1}, \Delta_{n} E_{1}^{2}, \ldots$ and $\Delta_{n} E_{2}^{1}, \Delta_{n} E_{2}^{2}, \ldots$ such that

$$
\Delta_{n} E_{1}^{1} \supseteq \Delta_{n} E_{2}^{2} \cup \Delta_{n} E_{2}^{3} \cup \cdots \cup \Delta_{n} E_{2}^{r} .
$$

where $r=r\left(E_{2}\right)$.

Using the lemma of [6] (see also [18, Lemma 7.5]), suitably adapted to the present setting, we deduce that the number $R_{n}$ of encounter zones within $B^{n}$ satisfies $R_{n} \leq\left|\Delta B^{n}\right|$. Taking expectations, we obtain from (3.5) that

$$
\mathbb{P}\left(R_{n}\right)=\eta(2 n-2 N+1)^{d} \leq\left|\Delta B^{n}\right| \leq 2 d^{2}(2 n)^{d-1},
$$


which is impossible for large $n$. We deduce by contradiction that $\mathbb{P}(M \geq 3)=0$ as required.

Proof of Theorem 3.4. A closely related proof may be found in [21]. Let $p_{\mathrm{rw}}, \eta>0$, and let $D_{N}$ be the event that $B^{N}$ contains no reflectors other than crossings. Then

$$
\mathbb{P}\left(D_{N}\right)=\left(p_{\mathrm{rw}}+p_{+}\right)^{(2 N)^{d}}
$$

Let $T$ be the minimum non-negative value of $m$ such that the point $(m, 0,0, \ldots, 0)$ is a rw point, and write $X=(T, 0,0, \ldots, 0)$. Since $p_{\text {rw }}>0$, we may choose an integer $t$ such that $\mathbb{P}(T \geq t) \leq \frac{1}{4} \eta$. Note that

$$
\mathbb{P}\left(T \geq t \mid D_{N}\right) \leq \mathbb{P}(T \geq t) \leq \frac{1}{4} \eta \text { for all } N
$$

by the FKG inequality.

Let $y \in \partial B^{N}$ satisfy $y=\left(y_{1}, y_{2}, y_{3}, \ldots, y_{d}\right) \neq( \pm N, 0,0, \ldots, 0)$. For $k \in\{-N,-N+$ $1, \ldots,-1\} \cup\{T+1, T+2, \ldots, N-1\}$, let $S_{k}$ be the set of points $(k, 0,0, \ldots, 0),\left(k, y_{2}, 0, \ldots, 0\right)$, $\left(k, y_{2}, y_{3}, 0, \ldots, 0\right), \ldots,\left(k, y_{2}, y_{3}, \ldots, y_{d}\right)$. Since $S_{k} \cap S_{l}=\varnothing$ if $k \neq l$, the events $U_{k}=$ \{all points in $S_{k}$ are rw points\} are conditionally independent given $D_{N}$ and the choice of $X$; furthermore, the conditional probability of $U_{k}$ is at least $p_{\mathrm{rw}}^{d}$, since $\left|S_{k}\right| \leq d$ for all $k$. If $U_{k}$ occurs for some $k$, and also $D_{N}$ and $\left\{T<\frac{1}{2} N\right\}$, then $X \leftrightarrow_{N} y$.

Now, if $t<\frac{1}{2} N$,

$$
\begin{aligned}
\mathbb{P}\left(X \varkappa_{N} y \mid T<t, D_{N}\right) \geq \mathbb{P}\left(\bigcup_{k} U_{k} \mid T<t, D_{N}\right) \\
=1-\prod_{\substack{k:-N \leq k<0 \\
\text { or } t \leq k<N}}\left(1-\mathbb{P}\left(U_{k} \mid D_{N}\right)\right) \\
\geq 1-\left(1-p_{\mathrm{rw}}^{d}\right)^{2 N-t} .
\end{aligned}
$$

We required above that $y \neq( \pm N, 0,0, \ldots, 0)$. It is immediate however that $X \leftrightarrow N$ $( \pm N, 0,0, \ldots, 0)$ so long as $T<t<\frac{1}{2} N$ and $D_{N}$ occurs.

There are at most $(2 N)^{d}$ possible choices for $y$. It follows that, for $t<\frac{1}{2} N$,

$$
\mathbb{P}\left(\text { for all } y \in \partial B^{N}, X \rightsquigarrow N y \mid T<t, D_{N}\right) \geq 1-(2 N)^{d}\left(1-p_{\text {rw }}^{d}\right)^{2 N-t} .
$$

Therefore, for $t<\frac{1}{2} N$,

$$
\mathbb{P}\left(B^{N} \text { is not good }\right) \leq \frac{1}{4} \eta+(2 N)^{d}\left(1-p_{\mathrm{rw}}^{d}\right)^{2 N-t}+1-\left(p_{\mathrm{rw}}+p_{+}\right)^{(2 N)^{d}}
$$

We pick $M(>2 t)$ such that $(2 N)^{d}\left(1-p_{\text {rw }}^{d}\right)^{2 N-t}<\frac{1}{4} \eta$ for $N \geq M$, and $A=A\left(p_{\text {rw }}\right)$ such that $1-(1-A)^{(2 M)^{d}}<\frac{1}{2} \eta$. The required conclusion follows.

Proof of Theorem 3.3. Let $p_{\mathrm{rw}}>0$. By Theorem 3.4, there exists $A=A\left(p_{\mathrm{rw}}\right)>0$ and an integer $M$ such that

$$
\mathbb{P}\left(B^{M} \text { is good }\right)>p_{\mathrm{c}}\left(\mathbb{L}^{2}, \text { site }\right),
$$


the critical probability of site percolation on $\mathbb{L}^{2}$. We choose $A$ and $M$ accordingly, and suppose for the moment that $1-p_{\mathrm{rw}}-p_{+}<A$. With $\gamma=\mathbb{P}\left(B^{M}\right.$ is good $)$, let $\theta(\gamma)$ be the percolation probability of site percolation on $\mathbb{L}^{2}$ with density $\gamma$. By the remarks after the statement of Theorem 3.4, and the fact that $\theta(\gamma)>0$, we have that there exists a.s. an infinite cluster of green sites in the renormalised lattice. Every corresponding box of the labyrinth contains some rw point, and all such rw points lie in the same equivalence class. Therefore, the labyrinth contains a.s. an infinite equivalence class. It follows that $\mathbb{P}(|C(0)|=\infty)>0$.

Assume now that $d \geq 3$. We shall use a slab argument, related to that used to prove Theorem 6.48 of [17]. We build the set $\bar{C}(0)$ in a natural recursive manner. Let $e_{1}, e_{2}, \ldots$ be a fixed ordering of the edges of $\mathbb{L}^{d}$, and suppose that 0 is a rw point. We select the earliest edge, $e$ say, which is incident to 0 , and we add, vertex by vertex, the unique light path departing 0 in the direction $e$. Having completed this step, and obtained a pair $\{0, x\}$ of (possibly identical) rw points, we pick the earliest edge incident with 0 or $x$ which has not yet been traversed, and we iterate the procedure. Continuing likewise until all possibilities have been exhausted, we have constructed the set $\bar{C}(0)$.

For $k \geq 1$, let $L_{k}$ be the region of $\mathbb{Z}^{d}$ containing all vertices $v=\left(v_{1}, v_{2}, \ldots, v_{d}\right)$ satisfying $2 k M<v_{1} \leq 2(k+1) M$, and let $H_{k}$ be the set of all $v$ with $v_{1}=k$. If $\bar{C}(0)$ contains some point $w=\left(w_{1}, w_{2}, \ldots, w_{d}\right)$ with $w_{1}>2 K M$, then $\bar{C}(0)$ contains some path traversing every 'slab' $L_{0}, L_{1}, \ldots, L_{K-1}$. Now, by Theorem 3.4 and the remarks thereafter, each time that the above construction encounters a new slab $L_{k}$ for the first time, there is probability at least $\theta(\gamma)$ that it intersects an infinite set of intercommunicating rw points contained in $L_{k}$. It follows (by the argument given in [17, pp. 127-128]) that

$$
\mathbb{P}^{*}\left(\bar{C}(0) \cap H_{2 K M+1} \neq \varnothing,|\bar{C}(0)|<\infty\right) \leq(1-\theta(\gamma))^{K} .
$$

Therefore

$$
\mathbb{P}^{*}(k \leq \operatorname{rad}(\bar{C}(0))<\infty) \leq 2 d(1-\theta(\gamma))^{\lfloor(k-1) /(2 M)\rfloor}
$$

and the theorem is proved (when $d \geq 3$ ).

We next present a sketch of the argument required when $d=2$. For $l=\left(l_{1}, l_{2}\right)$, let $B_{l}^{M}$ be the box $2 M l+B^{M}$, where $M$ is chosen to satisfy (3.6). We colour $l$ green if $B_{l}^{M}$ is a good box, and red otherwise. Using (3.6), there exists a.s. a unique infinite cluster $I$ of green vertices in the renormalised copy of $\mathbb{L}^{2}$ obtained by replacing each $B_{l}^{M}$ by the vertex $l$. Let $\mathcal{L}$ be the set of all $l$ such that $B_{l}^{M}$ intersects $\bar{C}(0)$. If $k \leq \operatorname{rad}(\bar{C}(0))<\infty$, then $\mathcal{L}$ contains a path of $\mathbb{L}^{2}$ whose endpoints are at least distance $\lfloor k /(2 M)\rfloor$ apart (in the norm $|\cdot|$ ), and in addition every $B_{l}^{M}$, for $l \in \mathcal{L}$, either is red, or is green but lies in a finite green (site) cluster of $\mathbb{L}^{2}$. Therefore, the renormalised site 0 belongs to a 'hole' in $I$, this hole having diameter at least $\lfloor k /(2 M)\rfloor$. The boundary of this hole contains a red circuit of $\mathbb{L}_{*}^{2}$, the matching lattice obtained from $\mathbb{L}^{2}$ by adding diagonals to each face; this red circuit has length at least $\lfloor k /(2 M)\rfloor$, and the origin lies in either the circuit or its interior. Using standard arguments from percolation theory (see [23, eqn 3.89], [17, Sect. 9.4]), we deduce that the probability of such a red circuit decays exponentially in $k /(2 M)$, so long as $M$ has been chosen sufficiently large that (3.6) holds.

Suppose now that $p_{\text {rw }}>p_{\mathrm{c}}$; we have from the definition of $p_{\mathrm{c}}$ that $\mathbb{P}^{*}(|C(0)|=\infty)>0$. Assume that $d \geq 3$, and let $p_{\mathrm{c}}(M)$ be the critical probability of site percolation on the slab $L^{M}=\left\{v \in \mathbb{Z}^{d}: 0<v_{1} \leq M\right\}$. We pick $M$ such that $p_{\text {rw }}>p_{\mathrm{c}}(M)$. [This may be done since $p_{\mathrm{c}}(M) \downarrow p_{\mathrm{c}}$ as $M \rightarrow \infty$. See $[18,20]$.] We now repeat the slab argument presented above, and (3.3) follows immediately. 
Finally consider the case when $p_{\mathrm{rw}}>p_{\mathrm{c}}$ and $d=2$. Let $A_{n}$ be the event that the annulus $[-2 n, 2 n]^{2} \backslash[-n, n]^{2}$ contains a circuit $C_{n}$ of rw points having the origin in its interior and such that $C_{n}$ lies in an infinite connected cluster of rw points. On $A_{n}$, we have that either $|C(0)|=\infty$ or $\operatorname{rad}(\bar{C}(0))<2 n$. However, $\mathbb{P}^{*}\left(A_{n}\right) \geq 1-e^{-n \xi}$ for some constant $\xi=\xi\left(p_{\text {rw }}\right)>0$, whence (3.3) follows.

In order to obtain the above bound for $\mathbb{P}^{*}\left(A_{n}\right)$, we may use standard path-intersection arguments from percolation; see $[17,23]$. Briefly, if the annulus fails to contain a circuit of $\mathrm{rw}$ points, then it is traversed in the matching lattice by a path of non-rw points. If such a circuit exists but is not in an infinite cluster, then its external boundary corresponds to a circuit of non-rw points in the matching lattice. Each of these two events has a probability which decays exponentially quickly to 0 as $n \rightarrow \infty$.

\section{An electrical network}

In the proof of Theorem 2.1, we shall require an estimate of the conductance of a certain electrical network arising from the labyrinth. Such an estimate is necessary in proving that the diffusion constants in Theorems 2.1 and 2.2 are strictly positive. The required notation and estimate are presented in this section.

Let $\omega \in \Omega$ and let $N$ be a positive integer. Write $B^{N}=[-N, N-1]^{d} \subseteq \mathbb{Z}^{d}$ as usual. The pair $(\omega, N)$ gives rise to a periodic configuration $\omega^{N}(\in \Omega)$ obtained by tiling $\mathbb{Z}^{d}$ with copies of $\omega$ restricted to $B^{N}$. More precisely, we first write $x \sim y$ if $x_{i}=y_{i} \bmod 2 N$ for $1 \leq i \leq d$. For each $y \in \mathbb{Z}^{d}$, we find the unique $x \in B^{N}$ satisfying $y \sim x$, and we set $\omega^{N}(y)=\omega(x)$. We denote by $[x]$ the equivalence class of a vertex $x$ under the equivalence relation $\sim$.

In advance of studying the labyrinth generated by $\omega^{N}$, we need to eliminate certain bad configurations. Suppose that $p_{\text {rw }}>0$. Let $0<\rho<1$, and consider the set of light paths of $\omega^{N}$. Let $\Omega^{N}=\Omega^{N, \rho}$ be the set of all $\omega(\in \Omega)$ for which every light path of $\omega^{N}$ has length not exceeding $N^{\rho}$. We shall see at (4.7) that

$$
\mathbb{P}\left(\Omega^{N}\right) \geq 1-c N^{d}\left(1-p_{\mathrm{rw}}\right)^{\frac{1}{2}\left\lfloor N^{\rho}\right\rfloor / d}
$$

where $c=c(d)$ is a positive constant. Thus $\mathbb{P}\left(\Omega^{N}\right)$ is close to 1 for large $N$, and we shall assume henceforth that the event $\Omega^{N}$ occurs, i.e., that

$$
\omega \in \Omega^{N}
$$

We shall concentrate on the set of rw points lying in $B^{N}$, and write $\mathcal{W}^{N}(\omega)=\mathcal{W}(\omega) \cap B^{N}$ $\left(=\mathcal{W}\left(\omega^{N}\right) \cap B^{N}\right)$ for this set. It will be useful to represent $\mathcal{W}^{N}$ as the set of nodes of an electrical network (illustrated in Figure 4). The 'terminals' of this network will be sets of nodes near to the 'left' and 'right' faces of $B^{N}$. We argue as follows in order to construct these sets.

Suppose that $x, y \in \mathcal{W}^{N}(\omega)$ are such that $n^{\omega^{N}}([x],[y])>0$. Pick $u \in[x], v \in[y]$ such that $n^{\omega^{N}}(u, v)>0$, and let $\pi$ be a corresponding light path of $\omega^{N}$, directed from $u$ to $v$. Let us keep count of the number of times $\pi$ intersects the set $\left\{x \in \mathbb{R}^{d}: x_{1} \in(2 \mathbb{Z}+1) N-\frac{1}{2}\right\}$; each time $\pi$ intersects this set in the direction of increasing first coordinate (resp. decreasing first coordinate) we score +1 (resp. -1 ). We write $s(\pi ; u, v)$ for the total score. Using the assumption that $\omega \in \Omega^{N}$, we may see that $s(\pi ; u, v)$ can take only the values $0, \pm 1$, and 


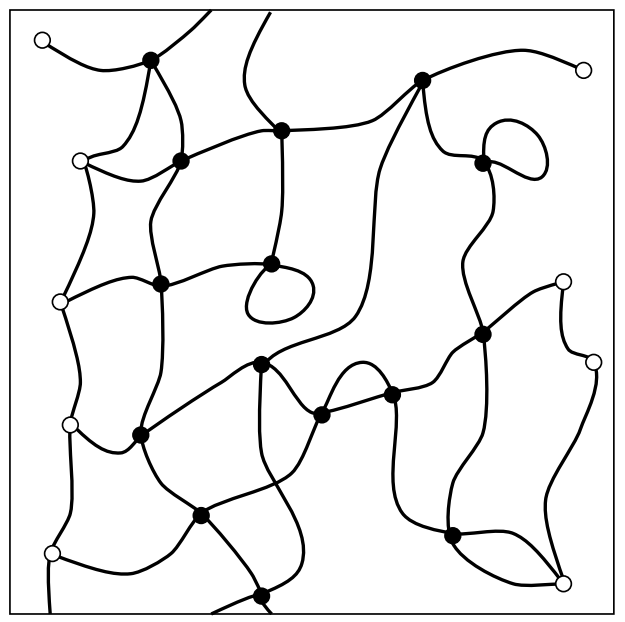

Fig. 4. An illustration of the electrical network constructed from an equivalence class $\mathcal{C}$ which straddles $B^{N}$. The open circles o represent rw points in the left and right 'terminals', and the dense circles • represent other rw points.

furthermore that $s(\pi ; u, v)$ depends only on $x$ and $y$, and not further on the choice of $u, v$, $\pi$. We define $m(x, y)=s(\pi ; u, v)$, and note that $m(x, y)=-m(y, x)$.

For $x, y \in \mathcal{W}^{N}(\omega)$ satisfying $n^{\omega^{N}}([x],[y])=0$, we define $m(x, y)=0$. Note that the function $m$ depends on $N$ and $\omega$, and we sometimes write $m=m^{N, \omega}$.

Suppose $x, y \in \mathcal{W}^{N}(\omega)$. We write $x \leftrightarrow_{N} y$ if $x \leftrightarrow^{\omega^{N}}[y]$. It is easily checked that $\leftrightarrow_{N}$ is an equivalence relation on $\mathcal{W}^{N}(\omega)$. Let $\mathcal{C}$ be an equivalence class of $\leftrightarrow_{N}$. We define the 'right edge' $r$ and 'left edge' $l$ of $\mathcal{C}$ as follows:

$$
\begin{aligned}
l=l^{N, \omega, \mathcal{C}} & =\{x \in \mathcal{C}: \exists y \in \mathcal{C} \text { with } m(x, y)=-1\}, \\
r=r^{N, \omega, \mathcal{C}} & =\{x \in \mathcal{C}: \exists y \in \mathcal{C} \text { with } m(x, y)=1\}, \\
\mathcal{M}=\mathcal{M}^{N, \omega, \mathcal{C}} & =\mathcal{C} \backslash(l \cup r) .
\end{aligned}
$$

Under (4.2), we have that $l \cap r=\varnothing$ for large $N$; note that $l$ and $r$ may be empty in general, but that $l=\varnothing$ if and only if $r=\varnothing$. We shall consider in Section 6 a Markov chain on the state space obtained from $\mathcal{C}$ by identifying all elements of $l$ and all elements of $r$. To that end, we define

$$
\mathcal{V}=\mathcal{V}^{N, \omega, \mathcal{C}}=\mathcal{M} \cup\{r\} \cup\{l\} .
$$

(We use the notation $l$ (resp. $r$ ) to denote both a subset of $\mathcal{C}$ and an element of $\mathcal{V}$. The cardinality of a set $A$ of vertices will be denoted by $|A|$.) A member of $\mathcal{V}$ is called a simple state if it is a singleton and a composite state otherwise.

Let $\mathcal{C}$ be an equivalence class of rw points of $B^{N}$ under the relation $\leftrightarrow_{N}$, as above. Assume that $r \neq \varnothing$ (and hence $l \neq \varnothing$ ) and that there exists a sequence $v_{0}, v_{1}, \ldots, v_{n}$ in $\mathcal{W}\left(\omega^{N}\right) \cap B^{N}$ such that

(i) $v_{0} \in l, v_{n} \in r, v_{i} \in \mathcal{M}$ for $1 \leq i<n$,

(ii) $v_{i-1} \leftrightarrow_{N} v_{i}$ for $i=1, \ldots, n$,

(iii) $n \geq 2$.

Under these circumstances, we say that the equivalence class $\mathcal{C}$ straddles $B^{N}$.

In studying the Markov chain on the state space $\mathcal{V}$, we shall interpret $\mathcal{V}$ as the node-set of a certain electrical network, and we shall estimate the conductance of this network between 
the composite nodes $l$ and $r$. The edges of the network are placed as follows. For $a, b \in \mathcal{V}$, with $\{a, b\} \neq\{l, r\}$, we place exactly $\kappa^{N}(a, b)$ unit resistors in parallel between $a$ and $b$, where the symmetric function $\kappa^{N}=\kappa^{N, \omega, \mathcal{C}}$ is given by

$$
\kappa^{N}(a, b)= \begin{cases}0 & \text { if }\{a, b\}=\{l, r\} \\ n^{\omega^{N}}(a,[b]) & \text { otherwise. }\end{cases}
$$

(Recall that the composite nodes $l$ and $r$ are subsets of $\mathbb{Z}^{d}$.)

Suppose that $\mathcal{C}$ is such that $l$ and $r$ are non-empty and disjoint. We have constructed a certain electrical network between the two 'terminals' $l$ and $r$, and this network has a certain effective conductance denoted as $\sigma^{N}=\sigma^{N, \omega, \mathcal{C}}$. We shall prove a theorem concerning the asymptotics of $\sigma^{N}$ as $N \rightarrow \infty$, for a suitably chosen equivalence class $\mathcal{C}$ of $\omega^{N}$. In advance of stating this theorem, we present some further notation.

Let $\mathcal{C}_{1}, \mathcal{C}_{2}, \ldots, \mathcal{C}_{s}$ be the equivalence classes of $\mathcal{W}^{N}$ under $\leftrightarrow_{N}$. Let $\mathcal{N}=\mathcal{C}_{J}$ be an equivalence class having maximal cardinality (if there are two or more such classes, we pick one according to some predetermined rule).

Theorem 4.1. Suppose that $p_{\mathrm{rw}}>0$ and $0<\rho<1$. Let $A$ be given as in Theorem 3.4 with $(1-\eta)^{2}=p_{\mathrm{c}}\left(\mathbb{L}^{2}\right.$, site $)$, and suppose that either $1-p_{\mathrm{rw}}-p_{+}<A$ or $p_{\mathrm{rw}}>p_{\mathrm{c}}$. There exist strictly positive constants $c_{i}$ such that the following statements are (simultaneously) valid with $\mathbb{P}$-probability approaching 1 as $N \rightarrow \infty$.

(a) The event $\Omega^{N}=\Omega^{N, \rho}$ occurs.

(b) We have that $|\mathcal{N}| \geq c_{1} N^{d}$.

(c) The sets $l=l^{N, \omega, \mathcal{N}}, r=r^{N, \omega, \mathcal{N}}$ are non-empty and disjoint, and satisfy $n^{\omega^{N}}(l, r) \geq$ $c_{2} N^{d-1}$.

(d) $\mathcal{N}$ is the unique equivalence class of $B^{N}$, under $\leftrightarrow_{N}$, which straddles $B^{N}$.

(e) The conductance $\sigma^{N}=\sigma^{N, \omega, \mathcal{N}}$ satisfies $\sigma^{N} \geq c_{3} N^{d-2}$.

Before proving this, we make a remark concerning the value of $A$. We have not attempted to 'maximise' this value in Theorem 4.1. A first step in this direction would be to note that the conclusion of the theorem is valid with $A$ replaced by the quantity $A^{\prime}$ given as in Theorem 3.4 with $1-\eta=p_{\mathrm{c}}\left(\mathbb{L}^{2}\right.$, site $)$. The proof of this stronger statement is slightly more complicated, and follows well trodden paths of percolation theory.

Proof. Assume that $p_{\text {rw }}>0$. Theorem 3.2 is not quite sufficient to imply that $\mathbb{P}\left(\Omega^{N}\right) \rightarrow 1$ as $N \rightarrow \infty$, since $\Omega^{N}$ is defined in terms of the periodic configuration $\omega^{N}$. However, the argument of the proof may be used to obtain the following conclusion. Let $\lambda^{N}(e)$ be the number of edges in the light path of $\omega^{N}$ containing the edge $e$, and let $R_{e}$ be the event that at least one endvertex of $e$ is a rw point. Then

$$
\mathbb{P}\left(R_{e}, \lambda^{N}(e)>k\right) \leq\left(1-p_{\text {rw }}\right)^{k /(2 d)} \quad \text { for } 0 \leq k<2 N,
$$

where we have used the fact that no light path of length less than $2 N$ can visit any equivalence class $[x]$ at two or more different vertices. Therefore

$$
\mathbb{P}\left(\Omega^{N}\right) \geq 1-2 d\left|B^{N}\right|\left(1-p_{\text {rw }}\right)^{\left\lfloor N^{\rho}\right\rfloor /(2 d)}
$$

as required for (4.1).

Assume now that $1-p_{\text {rw }}-p_{+}<A$, where $A$ is given in Theorem 3.4. Let $M$ be the integer given in the conclusion of Theorem 3.4. Following the discussion after that theorem, 
we colour the vertex $v \in \mathbb{Z}^{d}$ green if the translate $B_{v}^{M}=2 M v+B^{M}$ is good, and red otherwise. Since the colours of vertices are independent, and since

$$
\mathbb{P}(v \text { is green })>\sqrt{p_{\mathrm{c}}\left(\mathbb{L}^{2}, \text { site }\right)}>p_{\mathrm{c}}\left(\mathbb{L}^{2}, \text { site }\right) \quad \text { for } v \in \mathbb{Z}^{d},
$$

we deduce that there exists a.s. an infinite green cluster $\Gamma$ of $\mathbb{Z}^{d}$. By the definition of 'good', the corresponding region $\Gamma^{M}=2 M \Gamma+B^{M}$ is an infinite connected region of the labyrinth every rw point of which lies in some infinite equivalence class $\mathcal{E}$ of the labyrinth, and every other point of which belongs to some light path joining two rw points in $\mathcal{E}$. In particular, the labyrinth contains an infinite equivalence class $\mathcal{E}$, which by Theorem 3.1 is a.s. unique.

For notational convenience, we shall assume that $N=(2 k+1) M$ for some $k \geq 1$; equivalent arguments are valid without this assumption, but the notation becomes slightly more complicated. Under this assumption, $B^{N}$ may be partitioned into exactly $(2 k+1)^{d}$ translates of $B^{M}$, namely the set $2 M \mathcal{T}+B^{M}$ where $\mathcal{T}=[-k, k]^{d} \subseteq \mathbb{Z}^{d}$.

For positive constants $\mathbf{d}=\left(d_{i}\right)$, let $T_{k}(\mathbf{d})$ denote the intersection of the following events (i)-(iii).

(i) After appropriate re-labelling, the green clusters $\Gamma_{1}, \Gamma_{2}, \ldots$ of $\mathcal{T}$ satisfy $\left|\Gamma_{1}\right| \geq d_{1} k^{d}$, $\left|\Gamma_{i}\right| \leq d_{2} \log k$ for $i \neq 1$.

(ii) There exist at least $d_{3} k^{d-1}$ pairs of points in $\Gamma_{1}$ of the form $\left(-k, t_{2}, t_{3}, \ldots, t_{d}\right)$, $\left(k, t_{2}, t_{3}, \ldots, t_{d}\right)$; we denote by $L$ (resp. $R$ ) the set of all points $t$ lying in this family which satisfy $t_{1}=-k$ (resp. $t_{1}=k$ ).

(iii) There exist at least $d_{4} k^{d-1}$ site-disjoint paths of $\Gamma_{1}$ each joining some site of $L$ to some site of $R$.

There exist positive constants $\mathbf{d}=\left(d_{i}\right)$ such that

$$
\mathbb{P}\left(T_{k}(\mathbf{d})\right) \rightarrow 1 \quad \text { as } k \rightarrow \infty .
$$

The principal arguments necessary to establish this are fairly standard in percolation theory, and may be found in $[17,18]$. We therefore omit a full proof, and choose $\mathbf{d}$ accordingly. Here are some brief notes concerning parts (ii) and (iii). Let $\gamma=\mathbb{P}(0$ is green), and note that $\gamma^{2}>p_{\mathrm{c}}\left(\mathbb{L}^{2}\right.$, site). Let $I_{t}$ be the indicator function of the event $\{t$ is green $\}$. For $t=$ $\left(t_{1}, t_{2}, \ldots, t_{d}\right) \in \mathcal{T}$, let $g(t)=\left(-t_{1}, t_{2}, \ldots, t_{d}\right)$. Now define $J_{t}=I_{t} I_{g(t)}$, noting that $J_{t}=I_{t}$ if $t_{1}=0$, and that $J_{t}$ dominates a site percolation process on the subset $\Lambda=[-k, 0] \times[-k, k]^{d-1}$ of $\mathcal{T}$ having intensity $\gamma^{2}$. We call $t(\in \mathcal{T})$ black if $J_{t}=1$. Let $\varepsilon>0$. Using arguments of [20], we find that there exists a constant $d_{3}$ such that $\Lambda$ is traversed (in the short direction) by at least $d_{3} k^{d-1}$ site-disjoint black paths, with probability at least $1-\varepsilon$. Since $J_{t}=J_{g(t)}$, the reflections of these paths in the hyperplane $H_{0}=\left\{t \in \mathcal{T}: t_{1}=0\right\}$ are black also. Therefore, on the above event, $\mathcal{T}$ is traversed by at least $d_{3} k^{d-1}$ site-disjoint black paths with the following property: the two endpoints of each such path have the form $\left(-k, t_{2}, t_{3}, \ldots, t_{d}\right)$, $\left(k, t_{2}, t_{3}, \ldots, t_{d}\right)$ for some $t_{2}, t_{3}, \ldots, t_{d}$. Properties (ii) and (iii) follow, since $J_{t} \leq I_{t}$.

On the event $T_{k}(\mathbf{d})$, we have that $B^{N}$ contains an equivalence class $\mathcal{C}$ which includes all rw points in the region $2 M \Gamma_{1}+B^{M}$. Using the definition of 'good', this region contains at least $\left|\Gamma_{1}\right|$ rw points, whence $T_{k}(\mathbf{d})$ implies statement (b) of the theorem with an appropriate $c_{1}=c_{1}\left(d_{1}, M\right)>0$.

Clearly $\mathcal{C}$, defined above, straddles $B^{N}$. Next we prove that (with large probability) no other equivalence class $\mathcal{C}_{i}$ straddles $B^{N}$, and that $\mathcal{C}=\mathcal{N}$. There is a variety of ways of doing this, of which we choose a simple one. Consider the event that the following four statements hold:

- the vertex $x \in B^{N}$ is a rw point,

- $x \in l^{N, \omega, \mathcal{D}}$ for some equivalence class $\mathcal{D}$ of $\leftrightarrow_{N}$, 
- $\mathcal{D}$ straddles $B^{N}$

- $\Omega^{N}$ occurs.

Suppose we 'grow' $\mathcal{D}$ in a recursive way starting from $x$ (similar to the general method given in the proof of Theorem 3.3). Since $\mathcal{D}$ straddles $B^{N}$, and since $\Omega^{N}$ occurs, this construction must intersect every slab of the form $S_{r}=2 M T_{r}+B^{M}$, where $T_{r}=\left\{t \in \mathcal{T}: t_{1}=r\right\}$ and $r$ satisfies

$$
-N+N^{\rho} \leq 2 M r-M<2 M r+M \leq N-N^{\rho} .
$$

In the construction of $\mathcal{D}$, there occurs a first time that the light enters each $S_{r}$. On entering $S_{r}$, there is a strictly positive probability $\theta_{M}$ that $\mathcal{D}$ enters some translate $2 M t+B^{M}$ of $B^{M}$, with $t \in T_{r}$, such that $t$ is green and $t$ lies in a green cluster of $T_{r}$ having cardinality at least $d_{5} N^{d-1}$ for some $d_{5}>0$. On the event $T_{k}(\mathbf{d})$, this green cluster must, by virtue of its size (see (i) above), be a subset of $\Gamma_{1}$. Using the usual slab argument (see [17, pp. 127-128]), we deduce that, for an appropriate constant $c$,

$$
\mathbb{P}\left(\Omega^{N} \cap T_{k}(\mathbf{d}) \cap E_{x}\right) \leq c\left(1-\theta_{M}\right)^{\left\lfloor\left(N-N^{\rho}\right) / M\right\rfloor},
$$

where $E_{x}$ is the event that $x$ is a rw point belonging to an equivalence class other than $\mathcal{C}$ which straddles $B^{N}$. Therefore

$$
\mathbb{P}\left(\Omega^{N} \cap T_{k}(\mathbf{d}) \cap\left(\bigcup_{x} E_{x}\right)\right) \leq c(2 N)^{d-1} N^{\rho}\left(1-\theta_{M}\right)^{\left\lfloor\left(N-N^{\rho}\right) / M\right\rfloor} .
$$

This implies that

$$
\mathbb{P}\left(\bigcup_{x} E_{x}\right) \leq c(2 N)^{d-1} N^{\rho}\left(1-\theta_{M}\right)^{\left\lfloor\left(N-N^{\rho}\right) / M\right\rfloor}+\mathbb{P}\left(\overline{\Omega^{N}}\right)+\mathbb{P}\left(\overline{T_{k}(\mathbf{d})}\right) \rightarrow 0
$$

as $N \rightarrow \infty$. Therefore, with probability tending to 1 as $N \rightarrow \infty$, we have that $\mathcal{N}=\mathcal{C}$, and that no other equivalence class straddles $B_{N}$.

If $T_{k}(\mathbf{d}) \cap \Omega^{N}$ occurs, and in addition $\mathcal{C}=\mathcal{N}$, then statement (c) of the theorem follows by (ii) above. It remains to show (e). This we do in the way laid down in [19]. Let $l=l^{N, \omega, \mathcal{C}}$ and $r=r^{N, \omega, \mathcal{C}}$, and assume that $T_{k}(\mathbf{d}) \cap \Omega^{N}$ occurs.

Let $\pi_{1}, \pi_{2}, \ldots, \pi_{D}$ be a collection of site-disjoint green paths of $\Gamma_{1}$, each joining some vertex of $L$ to some vertex of $R$, where $D$ is maximal. We write $v_{i, j}, 1 \leq j \leq J_{i}$, for the green sites of $\pi_{i}$ taken in order, so that $v_{i, 1} \in L$ and $v_{i, J_{i}} \in R$. The corresponding translates $2 M v_{i, j}+B^{M}$ are good, and therefore each contains a seed. For each $v_{i, j}$ we pick a corresponding seed $s_{i, j} \in 2 M v_{i, j}+B^{M / 2}$. We think of the paths $\pi_{i}$ as joining the 'leftmost seeds' $L^{\prime}=\left\{s_{i, 1}: i=1,2, \ldots, D\right\}$ to the 'rightmost seeds' $R^{\prime}=\left\{s_{i, J_{i}}: i=1,2, \ldots, D\right\}$. Between any two consecutive seeds $s_{i, j}, s_{i, j+1}$ on any path $\pi_{i}$, there is an $\omega$-path of length not exceeding $2\left|B^{M}\right|=2(2 M)^{d}=S$, and therefore containing no more than $S$ rw points. The corresponding electrical path joining $s_{i, 1}$ to $s_{i, J_{i}}$ has resistance not exceeding $S\left(J_{i}-1\right)$.

Now each $s_{i, 1}$ (resp. $s_{i, J_{i}}$ ) is joined by some $\omega$-path to a member of $l$ (resp. $r$ ) lying in $2 M v_{i, 1}+B^{M}$ (resp. $\left.2 M v_{i, J_{i}}+B^{M}\right)$, this path containing fewer than $\frac{1}{2} S$ rw points. Using the Rayleigh monotonicity principle (see [13]), we deduce that the conductance $\sigma^{N}$ satisfies

$$
\sigma^{N} \geq \sum_{i=1}^{D} \frac{1}{S\left(J_{i}-1\right)+S}
$$

whence, by the arithmetic/harmonic mean inequality,

$$
\sigma^{N} \geq \frac{D^{2} / S}{\sum_{i=1}^{D} J_{i}} \geq \frac{\left(d_{4} k^{d-1}\right)^{2} / S}{(2 k+1)^{d}} \geq d_{6} k^{d-2} \geq d_{7} N^{d-2}
$$


for some strictly positive constants $d_{6}$ and $d_{7}$. Statement (e) of the theorem is therefore valid with probability tending to 1 as $N \rightarrow \infty$.

For the second part of the proof, we assume that $p_{\mathrm{rw}}>p_{\mathrm{c}}$. We write $\Lambda=[-N,-1] \times$ $[-N, N-1]^{d-1}$ and $R=[0, N-1] \times[-N, N-1]^{d-1}$ for the left and right parts of $B^{N}$, and $\Xi=[-2 N,-N-1] \times[-N, N-1]^{d-1}$. Then $A^{N}=\Xi \cup \Lambda$ is a copy of $B^{N}$. A left-right rw crossing of $\Lambda$ is a rw path of $\Lambda$ (i.e., a path all of whose vertices are rw points in $\Lambda$ ) whose endpoints $x$, $y$ satisfy $x_{1}=-N, y_{1}=-1$; we make a similar definition for top-bottom rw crossings of $\Lambda$, and also for crossings of other boxes in $\mathbb{Z}^{d}$.

For positive constants $\mathbf{d}=\left(d_{i}\right)$, let $U_{N}(\mathbf{d})$ be the intersection of the following events.

(i) $B^{N}$ contains a rw cluster (i.e., a connected subgraph of $\mathbb{L}^{d}$ all of whose vertices are rw points) of size at least $d_{1} N^{d}$, and no other rw cluster of size exceeding $\frac{1}{4} N$.

(ii) This large cluster contains at least $d_{2} N^{d-1}$ left-right rw crossings of $B^{N}$, and at least one top-bottom rw crossing of each of the sets $\Lambda$ and $R$.

(iii) $A^{N}$ contains at least $d_{2} N^{d-1}$ left-right rw crossings, in the configuration $\omega^{N}$.

Using standard arguments (see [1, 20,27]), there exist positive constants $\mathbf{d}$ such that $\mathbb{P}\left(U_{N}(\mathbf{d})\right) \rightarrow 1$ as $N \rightarrow \infty$, and we choose $\mathbf{d}$ accordingly.

On the event $\Omega^{N} \cap U_{N}(\mathbf{d})$, we write $\mathcal{C}$ for the large cluster under (i) above. Let $\mathcal{N}$ be the equivalence class of $\mathcal{W}^{N}$ under $\leftrightarrow^{N}$ which contains $\mathcal{C}$.

Assume that $\Omega^{N} \cap U_{N}(\mathbf{d})$ occurs. If $d=2$, then (ii) above precludes the existence of any other equivalence class of $\mathcal{W}^{N}$ which straddles $\Lambda$. When $d \geq 3$, we follow the argument which led to (4.9) in order to obtain the same conclusion. Let $p_{\mathrm{c}}(M)$ be the critical probability of site percolation on the slab $L^{M}=[0, M-2] \times \mathbb{Z}^{d-1}$. We choose $M$ such that $p_{\mathrm{rw}}>p_{\mathrm{c}}(M)$; this is possible since $p_{\mathrm{c}}(M) \rightarrow p_{\mathrm{c}}$ as $M \rightarrow \infty$, see [20]. Let $E_{x}$ be the event that $x$ is a rw point of $B^{N}$ which lies in an equivalence class other than $\mathcal{C}$ which straddles $\Lambda$. For $d_{3}>0$ and $x \in L^{M} \cap B^{N}$, we let $\theta_{M}(x)$ be the probability that $x$ lies in a rw cluster of $L^{M} \cap B^{N}$ having size at least $d_{3} N^{d-1}$. We pick $d_{3}(>0)$ in such a way that

$$
\theta_{M}=\inf \left\{\theta_{M}(x): x \in L^{M} \cap B^{N}\right\}>0 ;
$$

that this may be done is a consequence of the block construction of [20]. Now, as in (4.9),

$$
\mathbb{P}\left(\Omega^{N} \cap U_{N}(\mathbf{d}) \cap E_{x}\right) \leq c\left(1-\theta_{M}\right)^{\left\lfloor\left(N-N^{\rho}\right) / M\right\rfloor},
$$

for some $c>0$. Let $S^{N}(\Lambda)\left(\right.$ resp. $\left.S^{N}(R)\right)$ be the event that there exists no equivalence class other than $\mathcal{C}$ which straddles $\Lambda$ (resp. $R$ ). Arguing as following (4.9), we deduce that

$$
\mathbb{P}\left(\Omega^{N} \cap U_{N}(\mathbf{d}) \cap S^{N}(\Lambda) \cap S^{N}(R)\right) \rightarrow 1 \quad \text { as } N \rightarrow \infty,
$$

which implies the validity of (d). Using (i) and (iii), we deduce that claim (c) holds on the event $\Omega^{N} \cap U_{N}(\mathbf{d}) \cap S^{N}(\Lambda) \cap S^{N}(R)$.

It remains to establish claim (e), and to this end we employ a block argument. Here is some notation. Let $M$ be a positive integer. For $\alpha \in\{-,+\}$ and $1 \leq j \leq d$, we define the box

$$
J_{\alpha, j}= \begin{cases}{[-M, M-1]^{j-1} \times[-2 M,-M-1] \times[-M, M-1]^{d-j}} & \text { if } \alpha=- \\ {[-M, M-1]^{j-1} \times[M, 2 M-1] \times[-M, M-1]^{d-j}} & \text { if } \alpha=+,\end{cases}
$$

and the face

$$
F_{\alpha, j}= \begin{cases}{[-M, M-1]^{j-1} \times\{-2 M\} \times[-M, M-1]^{d-j}} & \text { if } \alpha=- \\ {[-M, M-1]^{j-1} \times\{2 M-1\} \times[-M, M-1]^{d-j}} & \text { if } \alpha=+,\end{cases}
$$




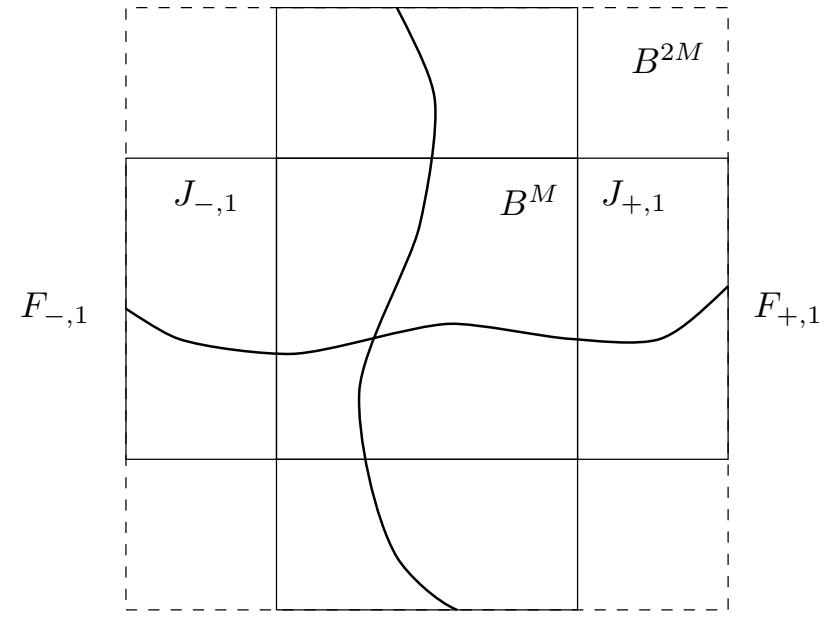

Fig. 5. The inner square $B^{M}$ has four 'ears' corresponding to the regions $J_{\alpha, j}$ for $\alpha \in\{-,+\}$ and $j=1,2$.

See Figure 5 for an illustration of this definition when $d=2$. We call the box $B^{2 M}$ good in $\omega$ if the following hold. [Here, $d_{4}$ is a positive constant to be chosen shortly.]

- $B^{M}$ contains a unique rw cluster of size at least $d_{4} M^{d}$, and no other rw cluster of size exceeding $\frac{1}{4} M$.

- For every $\alpha \in\{-,+\}$ and $j \in\{1,2, \ldots, d\}$, the region $B^{M} \cup J_{\alpha, j}$ contains a unique rw cluster $\mathcal{C}_{\alpha, j}$ of size at least $d_{4} M^{d}$, and no other rw cluster of size exceeding $\frac{1}{4} M$. Furthermore $\mathcal{C}_{\alpha, j}$ contains a vertex lying in the face $F_{\alpha, j}$.

Using arguments of percolation theory (see [1, 20, 27]), we may show the following. There exists a strictly positive constant $d_{4}$ such that: for all $\varepsilon>0$, there exists $M=M(\varepsilon)$ satisfying

$$
\mathbb{P}\left(B^{2 M} \text { is good }\right)>1-\varepsilon .
$$

We pick $d_{4}$ accordingly.

Any translate of $B^{2 M}$ is called good if the corresponding facts hold for that translate. We colour the vertex $v \in \mathbb{Z}^{d}$ blue if the translate $B_{v}^{2 M}=3 M v+B^{2 M}$ is good, and grey otherwise.

We now assume that $N=(3 k+2) M+1$ for some $k \geq 1$, noting that $B^{N-1}$ may be expressed as the union of the set $3 M \mathcal{T}+B^{2 M}$, where $\mathcal{T}=[-k, k]^{d} \subseteq \mathbb{Z}^{d}$. Unlike in the first part of this proof, these blocks may intersect one another; therefore the colours of the points in $\mathcal{T}$ are dependent random variables. The event $\{t$ is blue $\}$ depends on the states of vertices lying in the region

$$
S_{t}=3 M t+\left\{B^{M} \cup\left(\bigcup_{\substack{\alpha \in\{-,+\} \\ j \in\{1,2, \ldots, d\}}} J_{\alpha, j}\right)\right\} .
$$

Since $S_{v} \cap S_{w}=\varnothing$ if $|v-w| \geq 2$, these colours form a 1-dependent family of identically distributed random variables. Let $\pi$ satisfy $\sqrt{p_{\mathrm{c}}\left(\mathrm{site}, \mathbb{L}^{2}\right)}<\pi<1$. Using the main conclusion of [25], we may choose $\varepsilon$ in (4.12) sufficiently small to ensure that these colours dominate (stochastically) a site percolation process on $\mathcal{T}$ with density $\pi$.

We now follow the arguments presented after (4.8). Let $\Lambda_{k}$ be a set (with maximal cardinality) of site-disjoint blue paths traversing $\mathcal{T}$ between its left face $\{-k\} \times[-k, k]^{d-1}$ 
and its right face $\{k\} \times[-k, k]^{d-1}$, and having the property that the two endpoints $v$ and $w$ of every such path satisfy $v_{1}=-k, w_{1}=k, v_{j}=w_{j}$ for $2 \leq j \leq d$. Then

$$
\mathbb{P}\left(\left|\Lambda_{k}\right| \geq d_{5} k^{d-1}\right) \rightarrow 1 \quad \text { as } k \rightarrow \infty
$$

for some $d_{5}>0$.

Let $v$ and $w$ be blue neighbours in $\mathbb{L}^{d}$. Since $S_{v} \cap S_{w}$ is a box of dimensions $M \times 2 M \times$ $\cdots \times 2 M$ which is traversed by rw paths in its short direction, we find that the 'large' $r w$ clusters in these boxes form part of the same (larger) rw cluster in the union $S_{v} \cup S_{w}$. It follows that $B^{N-1}$ contains at least $\left|\Lambda_{k}\right|$ rw paths each of which has the property that its endpoints $x, y$ satisfy $x_{1}=-N+1, y_{1}=N-2,\left|x_{j}-y_{j}\right| \leq 4 M$ for $2 \leq j \leq d$.

For $t \in \mathcal{T}$, we define the 'left' and 'right' edges of $S_{t}$ by

$$
\begin{aligned}
& L_{t}=3 M t+F_{-, 1}-u_{1}=3 M t+\{-2 M-1\} \times[-M, M-1]^{d-1}, \\
& R_{t}=3 M t+F_{+, 1}+u_{1}=3 M t+\{2 M\} \times[-M, M-1]^{d-1} .
\end{aligned}
$$

[Here, $u_{1}$ is a unit vector in the direction of increasing first coordinate.] Let $V=\{v(\lambda)$ : $\left.\lambda \in \Lambda_{k}\right\}$ be the set of left endpoints $v(\lambda)$ of paths $\lambda$ in $\Lambda_{k}$, and $W=\left\{w(\lambda): \lambda \in \Lambda_{k}\right\}$ the set of right endpoints $w(\lambda)$. For $\lambda \in \Lambda_{k}$, we define $I_{\lambda}$ to be the indicator function of the event that all vertices in $L_{v(\lambda)} \cup R_{w(\lambda)}$ are rw points. Conditional on $\Lambda_{k}$, the $I_{\lambda}$ are identically distributed random variables with

$$
\mathbb{P}\left(I_{\lambda}=1\right)=p_{\mathrm{rw}}^{2(4 M)^{d-1}}>0 .
$$

Let $\Lambda_{k}$ be given. By Cramér's theorem or otherwise, there exist strictly positive constants $c_{i}$ such that

$$
\mathbb{P}\left(\sum_{\lambda \in \Lambda_{k}} I_{\lambda} \geq c_{2}\left|\Lambda_{k}\right| \mid \Lambda_{k}\right) \geq 1-\exp \left(-c_{3}\left|\Lambda_{k}\right|\right) .
$$

If $\lambda \in \Lambda_{k}$ and $I_{\lambda}=1$, then $\lambda$ gives rise to a left-right rw path of $B^{N}$ whose left and right endvertices $x, y$ satisfy $x_{j}=y_{j}$ for $2 \leq j \leq d$.

Suppose that the events in (4.11), (4.13), (4.14) occur. Combining the above observations, we conclude as in the first part of this proof that claim (e) is valid with probability approaching 1 as $N \rightarrow \infty$.

\section{Proof of Theorem 2.1}

Our principal method is the general invariance principle of Kipnis and Varadhan [24] and DeMasi, Ferrari, Goldstein, and Wick [11]; see also [16]. The proof that the diffusion constant is strictly positive is distinct from the application of the general principle, and is contained in Section 6. Since we shall make several appeals to this invariance principle, we begin by stating it (Theorem 5.1 below).

We suppose that $(\xi(n): n \geq 0)$ is a discrete-time Markov chain on a topological space $\Sigma$, which is ergodic and reversible with respect to a given invariant measure $\nu$. Let $F$ be a Borelmeasurable function from $\Sigma \times \Sigma$ to $\mathbb{R}^{d}$ which is anti-symmetric (i.e., $F\left(\omega, \omega^{\prime}\right)=-F\left(\omega^{\prime}, \omega\right)$ for all $\left.\omega, \omega^{\prime}\right)$. We define

$$
\begin{aligned}
I(n) & =F(\xi(n-1), \xi(n)), & & n \geq 1, \\
X(n) & =\sum_{k=1}^{n} I(k), & & n \geq 0, \\
X^{\varepsilon}(t) & =\varepsilon X\left(\left\lfloor\varepsilon^{-2} t\right\rfloor\right), & & \varepsilon>0, t>0 .
\end{aligned}
$$


Let $Q$ be the transition probability measure for the chain $\xi$. Then $Q$ may be regarded as an operator on $L^{2}(\Sigma, \mathcal{B}(\Sigma), \nu)$, where $\mathcal{B}(\Sigma)$ is the collection of Borel subsets of $\Sigma$, by defining

$$
Q f(\sigma)=\int f\left(\sigma^{\prime}\right) Q\left(\sigma, d \sigma^{\prime}\right)=E^{\sigma}(f(\xi(1)))
$$

for $f \in L^{2}(\Sigma, \mathcal{B}(\Sigma), \nu)$, where $E^{\sigma}$ is the law of $\xi$ when $\xi(0)=\sigma$. We define the vector

$$
\Phi(\sigma)=E^{\sigma}(I(1)) \text { for } \sigma \in \Sigma
$$

Finally, we write $\langle f(\xi)\rangle_{\nu}$ for the mean of a function $f$ of $\xi$, under the assumption that $\xi(0)$ has distribution $\nu$. That is to say

$$
\langle f(\xi)\rangle_{\nu}=\nu\left(E^{\sigma}(f(\xi))\right)
$$

for appropriate functions $f$. As usual, $L^{2}(\Sigma, \mathcal{B}(\Sigma), \nu)$ may be endowed with an inner product $\langle\cdot, \cdot\rangle_{\nu}$ by

$$
\langle f, g\rangle_{\nu}=\int f(\sigma) \overline{g(\sigma)} d \nu(\sigma)=\langle f(\xi(0)) \overline{g(\xi(0))}\rangle_{\nu}
$$

With this notation, we have that

$$
\left\langle f, Q^{n} g\right\rangle_{\nu}=\langle f(\xi(0)) g(\xi(n))\rangle_{\nu}
$$

for real-valued functions $f$ and $g$.

Theorem 5.1. [11] Assume that $\left\langle I(1)^{2}\right\rangle_{\nu}<\infty$. Let $\mathbf{D}$ be the real symmetric $d \times d$ matrix whose entries $D_{i j}$ are given by

$$
D_{i j}=\left\langle I_{i}(1) I_{j}(1)\right\rangle_{\nu}-2\left\langle\Phi_{i}(1-Q)^{-1} \Phi_{j}\right\rangle_{\nu}
$$

Then $X^{\varepsilon}$ converges $\nu$-dp to $\mathbf{D}^{1 / 2} W$ as $\varepsilon \downarrow 0$, where $W$ be a standard Brownian motion in $\mathbb{R}^{d}$, and $\mathbf{D}^{1 / 2}$ is a real symmetric square root of $\mathbf{D}$. Furthermore, if $u$ is a unit vector of $\mathbb{R}^{d}$, then

$$
\left\langle\left(u \cdot X^{\varepsilon}(t)\right)^{2}\right\rangle_{\nu} \rightarrow\left(u^{\prime} \mathbf{D} u\right) t \quad \text { as } \varepsilon \downarrow 0 .
$$

As before, we say that $X^{\varepsilon}$ converges $\nu$-dp to $\mathbf{D}^{1 / 2} W$ if

$$
E^{\sigma}\left(f\left(X^{\varepsilon}\right)\right) \rightarrow E\left(f\left(\mathbf{D}^{1 / 2} W\right)\right) \quad \text { in } \nu \text {-probability }
$$

for all bounded continuous functions $f$ on the appropriate space.

We shall make several applications of this theorem in proving Theorems 2.1 and 2.2. The basic method is similar to that used in $[11,24]$, namely to take $\Sigma=\Omega$, and to let $\xi(n)$ be the environment seen from the position of the light ray at its $n$th visit to the set $\mathcal{W}$ of rw points. We begin with an elementary lemma. We define translation operators on $\Omega$ as follows. For $x \in \mathbb{Z}^{d}$, let $\tau_{x}: \Omega \rightarrow \Omega$ be given by $\left(\tau_{x} \omega\right)_{y}=\omega_{y-x}$ for $y \in \mathbb{Z}^{d}$. We call an environment $\omega$ periodic if there exists $x(\neq 0)$ such that $\tau_{x} \omega=\omega$. The set of periodic configurations is denoted $\mathcal{T}$. We call the probability measure $\mu$ a point mass if there exists $\sigma \in \mathcal{R} \cup\{\varnothing\}$ such that $\mu(\{\sigma\})=1$. 
Lemma 5.2. If $\mu$ is not a point mass, then $\mathbb{P}(\mathcal{T})=0$.

Proof. Suppose that $\mu$ is not a point mass. Then

$$
\mathbb{P}(\mathcal{T}) \leq \sum_{x \in \mathbb{Z}^{d} \backslash\{0\}} \mathbb{P}(\omega(n x)=\omega(0) \text { for all } n)=0
$$

as required.

The only point mass which is consistent with the condition $p_{\mathrm{rw}}>0$ of Theorems 2.1 and 2.2 is the point mass on the state $\omega(x)=\varnothing$ for all $x$. For this environment, the chain $X^{\omega}$ is the usual symmetric random walk, for which the claims are well known. Therefore we may assume henceforth that $\mu$ is not a point mass.

In the light of Lemma 5.2, it suffices to prove a central limit theorem under the assumption that the environment space is $\widetilde{\Omega}=\Omega \backslash \mathcal{T}$. Suppose that 0 is a rw point. With $X^{\omega}(0)=0$ and $X^{\omega}$ defined as before (see equation $(2.1)$ ), we let $\xi(n)$ be the environment seen from the position $X^{\omega}(n)$, that is, $\xi(n)=\tau_{-x} \omega$ if $X^{\omega}(n)=x$. It may now be seen that $(\xi(n): n \geq 0)$ is a Markov chain on $\Omega^{*} \cap \widetilde{\Omega}$ with transition function

$$
Q\left(\omega, d \omega^{\prime}\right)=\frac{1}{2 d} \sum_{x \in \mathcal{W}(\omega)} n^{\omega}(0, x) \delta_{\tau_{-x} \omega}\left(d \omega^{\prime}\right),
$$

where $\delta_{\psi}$ is the probability measure on $\Omega$ which places a unit of probability on the environment $\psi$.

Define $F$ on $\widetilde{\Omega} \times \widetilde{\Omega}$ by

$$
F\left(\omega, \omega^{\prime}\right)= \begin{cases}x & \text { if } \omega^{\prime}=\tau_{-x} \omega \\ 0 & \text { otherwise }\end{cases}
$$

and let $(I(n): n \geq 0),(X(n): n \geq 0)$, and $\left(X^{\varepsilon}(t): t \geq 0\right)$ be given by (5.1)-(5.3), and $\Phi$ as in (5.5). Note that $X(n)=X^{\omega}(n)-X^{\omega}(0)$ where $\omega=\xi(0)$, and that $X^{\varepsilon}(t)=X^{\varepsilon, \omega}(t)$ for $\omega=\xi(0) \in \Omega^{*}$ (cf. (2.3) and the paragraph containing (2.1)).

It is now straightforward to verify that Theorem 5.1 may be applied to the process $\xi$ with either $\mathbb{P}^{*}$ or $\mathbb{P}^{* *}$ in the place of $\nu$. There follow some remarks about this.

In order to check that $\mathbb{P}^{*}$ and $\mathbb{P}^{* *}$ are reversible invariant measures for $\xi$, one need only check the detailed balance relation: for $A, B \in \mathcal{B}(\Omega)$, and for $P=\mathbb{P}^{*}, \mathbb{P}^{* *}$,

$$
\iint 1_{A}(\omega) 1_{B}\left(\omega^{\prime}\right) Q\left(\omega, d \omega^{\prime}\right) P(d \omega)=\iint 1_{A}(\omega) 1_{B}\left(\omega^{\prime}\right) Q\left(\omega^{\prime}, d \omega\right) P\left(d \omega^{\prime}\right) ;
$$

this may be checked directly from the definition (5.8) of $Q$.

The proof that $\mathbb{P}^{* *}$ is an ergodic invariant measure for the chain $\xi$ follows exactly the proof of the corresponding Lemma 5.9 in $[11$, p. 825]. In the present case, the argument uses the fact proved in Theorem 3.1 that any infinite equivalence class of rw points is a.s. unique.

The square integrability of $I(1)$ under both $\mathbb{P}^{*}$ and $\mathbb{P}^{* *}$ is a consequence of Theorem 3.2.

One may now apply Theorem 5.1 to the situation described in Theorem 2.1, thereby obtaining as weak limit a Wiener process having covariance matrix $\mathbf{D}$ given by (5.6). In order to show that $\mathbf{D}$ is diagonal, one proceeds just as in the proof of Theorem 5.6(iii) in [11, p. 823]. 
It follows that $\mathbf{D}=D \mathbf{I}$ where $\mathbf{I}$ is the identity matrix, and

$$
D=\left\langle i(1)^{2}\right\rangle_{\mathbb{P}^{* *}}-2\left\langle\phi(1-Q)^{-1} \phi\right\rangle_{\mathbb{P}^{* *}}
$$

where $i=I_{1}$ and $\phi=\Phi_{1}$ (the subscript indicates that we are taking the first coordinate). In the next section we prove that the constant $D$ is strictly positive, under the conditions appropriate for Theorem 2.1.

\section{Strict positivity of the diffusion constant}

This section is devoted to proving the following.

Lemma 6.1. Let $p_{\mathrm{rw}}>0$. There exists a strictly positive constant $A=A\left(p_{\mathrm{rw}}\right)$ such that $D>0$ whenever either $1-p_{\mathrm{rw}}-p_{+}<A$ or $p_{\mathrm{rw}}>p_{\mathrm{c}}$.

This we prove by the general route described for Theorem 5.7 in [11, p. 828 et seq.], and we begin with a sketch of the argument. Fix $\omega \in \Omega$. We shall approximate the process $X^{\omega}$ by another process (denoted $X^{N, \omega}$ ) which is constructed in a certain way on the periodic configuration $\omega^{N}$ (see Section 4). It will suffice to study the first coordinate $X_{1}^{N, \omega}$ of this process, and we shall study the asymptotic variance of $X_{1}^{N, \omega}$ by expressing it as an additive functional of a certain 'driving process' $\xi^{N, \omega}$. The latter process will be essentially a random walk on the set $\mathcal{W}^{N}(\omega)$ of rw points in the box $B^{N}$ endowed with periodic boundary conditions. This random walk will be reversible with respect to the uniform distribution on $\mathcal{W}^{N}$.

Taking the above paragraph on trust for the moment, we may see that the asymptotics of $X_{1}^{N, \omega}$ will be given in terms of the number of times that $\xi^{N, \omega}$ heads either eastwards from the right (hyper)edge of $B^{N}$, or westwards from the left (hyper)edge of $B^{N}$ (recall that we are regarding $B^{N}$ as a torus).

There are complications in pursuing this strategy. First, we need to 'recognise' the effect on $X^{N, \omega}$ of transitions of $\xi^{N, \omega}$. In order to do this, we shall assume that $\omega \in \Omega^{N} \cap \widetilde{\Omega}$, where $\Omega^{N}$ is defined above (4.1) with $\rho$ chosen to satisfy

$$
0<\rho<\frac{1}{d+3}
$$

The assumed absence of long light paths will be relevant to the relationship of $X^{N, \omega}$ to $\xi^{N, \omega}$.

Secondly, the process $\xi^{N, \omega}$ is not generally ergodic, since its state space $\mathcal{W}^{N}$ generally contains a multiplicity of closed sets. These sets are exactly the equivalence classes of the relation $\leftrightarrow_{N}$. Each such set $\mathcal{D}$ gives rise to a separate diffusion constant $D^{N, \omega, \mathcal{D}}$. We shall use Theorem 4.1 to show that $D^{N, \omega, \mathcal{D}}=0$ if $\mathcal{D}$ does not straddle $B^{N}$, and that there is (with probability tending to 1 as $N \rightarrow \infty$ ) a unique $\mathcal{D}$ which straddles $B^{N}$.

Let $\mathcal{N}$ denote this unique straddling set, when it exists. We shall (in Lemma 6.2) relate $D^{N, \omega, \mathcal{N}}$ to the conductance of $\mathcal{N}$ viewed as an electrical network (as in Section 4 ). In order to utilise the theory of electrical networks, we recall from Section 4 the identifications of certain sets of rw points near (respectively) the left and right edges of $B^{N}$. These identifications will contribute to the definition of the process $X^{N, \omega}$.

Let $D^{N, \omega}$ denote the asymptotic diffusion constant for the process $X_{1}^{N, \omega}$, suitably normalised. Using Fatou's lemma together with the formulae for $D$ and $D^{N}$, we shall obtain that

$$
D \geq \limsup _{N \rightarrow \infty} \mathbb{P}^{N}\left(D^{N}\right)
$$


where $\mathbb{P}^{N}$ denotes $\mathbb{P}$ conditioned on $\Omega^{N}$, i.e., $\mathbb{P}^{N}(\cdot)=\mathbb{P}\left(\cdot \mid \Omega^{N}\right)$.

We shall next express $D^{N, \omega}$ in terms of $D^{N, \omega, \mathcal{N}}$, and shall apply Theorem 4.1 in order to obtain that

$$
\mathbb{P}^{N}\left(D^{N} \geq c\right) \rightarrow 1 \quad \text { as } N \rightarrow \infty
$$

for some strictly positive constant $c$. It will follow by (6.2) that $D>0$.

\subsection{A Process on a STRIP}

Let $\rho$ satisfy (6.1), and let $\Omega^{N}$ be the set of all $\omega \in \Omega$ such that $\omega^{N}$ has no light path of length exceeding $N^{\rho}$. We have from Theorem 4.1(a) that $\mathbb{P}\left(\Omega^{N}\right) \rightarrow 1$ as $N \rightarrow \infty$. Assume for now that

$$
\omega \in \Omega^{N} \cap \widetilde{\Omega} .
$$

We shall work with the set $\mathcal{W}^{N}(\omega)$ of rw points lying in $B^{N}$, and we follow the notation of Section 4. As in that section, for any equivalence class $\mathcal{C}$ which straddles $B^{N}$, we define its left edge $l=l^{N, \omega, \mathcal{C}}$ and right edge $r=r^{N, \omega, \mathcal{C}}$ appropriately. With $\mathcal{M}=\mathcal{C} \backslash(l \cup r)$, we shall construct a Markov chain on the state space

$$
\mathcal{V}=\mathcal{V}^{N, \omega, \mathcal{C}}=\mathcal{M} \cup\{r\} \cup\{l\},
$$

and we shall explain how this Markov chain is related to the electrical network on $\mathcal{V}$ studied in Section 4.

We define the symmetric function $n^{N}=n^{N, \omega, \mathcal{C}}: \mathcal{V} \times \mathcal{V} \rightarrow \mathbb{R}$ by

$$
\begin{aligned}
n^{N}(l, r)=n^{N}(r, l) & =n^{\omega^{N}}(r,[l]), \\
n^{N}(v, w) & =n^{\omega^{N}}(v,[w]) \quad \text { if }\{v, w\} \neq\{r, l\},
\end{aligned}
$$

and the function $Q^{N}=Q^{N, \omega, \mathcal{C}}: \mathcal{V} \times \mathcal{V} \rightarrow \mathbb{R}$ by

$$
Q^{N}(v, w)=\frac{1}{2 d|v|} n^{N}(v, w) .
$$

(We recall some notation. First, $[w]=\left\{y \in \mathbb{Z}^{d}: y_{i}=w_{i} \bmod 2 N\right.$ for $\left.1 \leq i \leq d\right\}$, and $[l]=\left\{y \in \mathbb{Z}^{d}: y \in[w]\right.$ for some $\left.w \in l\right\}$. Secondly, $|v|=1$ if $v$ is a singleton, and otherwise $|v|$ is the cardinality of the subset $v$ of $B^{N}$.) Note that $n^{N}$ agrees with the function $\kappa^{N}$ defined in (4.5), except for the terms $n^{N}(r, l), n^{N}(l, r)$. Let $X^{N}=X^{N, \omega, \mathcal{C}}=\left(X^{N, \omega, \mathcal{C}}(n): n \geq 0\right)$ be the Markov chain with state space $\mathcal{V}+2 N \mathbb{Z} u_{1}$ (where $u_{1}$ is a unit vector in the direction of increasing first coordinate), and with transition probabilities given as follows. For $i, j \in \mathbb{Z}$, define $q^{N}=q^{N, \omega, \mathcal{C}}$ by

$$
\begin{aligned}
q^{N}\left(r+2 N i u_{1}, l+2 N j u_{1}\right) & =\delta_{i, j-1} Q^{N}(r, l), \\
q^{N}\left(l+2 N i u_{1}, r+2 N j u_{1}\right) & =\delta_{i, j+1} Q^{N}(l, r), \\
q^{N}\left(v+2 N i u_{1}, w+2 N j u_{1}\right) & =\delta_{i, j} Q^{N}(v, w), \quad \text { if }\{v, w\} \neq\{r, l\},
\end{aligned}
$$

where $\delta_{i, j}$ is the Kronecker delta. That is to say, $X^{N}$ is a random walk on the set $\mathcal{V}+2 N \mathbb{Z} u_{1}$. At each stage, the walker departs the current rw point in a random direction, and proceeds to the next rw point reached along the subsequent light path. The process $X^{N}$ is defined on the strip $B^{N}+2 N \mathbb{Z} u_{1}$. 
From the process $X^{N}$ we obtain a new process $\xi^{N}=\xi^{N, \omega, \mathcal{C}}$ by projecting $X^{N}$ onto $\mathcal{V}$. That is,

$$
\xi^{N}(n)=v \quad \text { if } \quad X^{N}(n)=v+2 N i u_{1} \text { for some } v \in \mathcal{V}, i \in \mathbb{Z} .
$$

It may be seen that $\xi^{N}$ is a Markov chain on $\mathcal{V}$ having transition probabilities $Q^{N}$ given by (6.7).

Let $\left(I^{N}(n): n \geq 0\right)=\left(I^{N, \omega, \mathcal{C}}(n): n \geq 0\right)$ be defined by:

$$
\begin{aligned}
& I^{N}(0)=0 \\
& I^{N}(n)=X_{1}^{N}(n)-X_{1}^{N}(n-1) \quad \text { if } n \geq 1
\end{aligned}
$$

where the subscript indicates that we are taking the first coordinate, and we adopt the convention that

$$
\left(r+2 N k u_{1}\right)_{1}=(1+2 k) N,\left(l+2 N k u_{1}\right)_{1}=(-1+2 k) N, \quad \text { for } k \in \mathbb{Z} .
$$

We have therefore that

$$
X_{1}^{N}(n)=\sum_{m=1}^{n} I^{N}(m)=\sum_{m=1}^{n} F^{N}\left(\xi^{N}(n-1), \xi^{N}(n)\right),
$$

where $F^{N}=F^{N, \omega, \mathcal{C}}$ is given by

$$
F^{N}(v, w)=w_{1}-v_{1}+2 N G^{N}(v, w) \quad \text { for } v, w \in \mathcal{V}
$$

and

$$
G^{N}(v, w)= \begin{cases}1 & \text { if }(v, w)=(r, l) \\ -1 & \text { if }(v, w)=(l, r) \\ 0 & \text { otherwise }\end{cases}
$$

Note that $F^{N}$ is anti-symmetric and bounded (for any given $N$ ).

We define a probability measure $\alpha^{N}=\alpha^{N, \omega, \mathcal{C}}$ on $\mathcal{V}$ by

$$
\alpha^{N}(u)=\frac{|v|}{|\mathcal{C}|}, \quad v \in \mathcal{V} .
$$

Then $\alpha^{N}$ is a reversible ergodic invariant measure for the chain $\xi^{N}$. Since $\omega \in \Omega^{N}$ (cf. (6.5)), the process $X_{1}^{N}$ can be realised via $(6.11)$ as an additive process in the sense of Theorem 5.1, with $\xi^{N}$ and $\alpha^{N}$ playing the roles of the driving process $\xi$ and invariant measure $\nu$ respectively, and with $F$ replaced by $F^{N}$.

We now apply Theorem 5.1 in this situation. The required square-integrability condition is satisfied, since $F^{N}$ is bounded. We deduce an invariance principle for the process $X_{1}^{N}$, suitably re-scaled, having some diffusion constant $D^{N}=D^{N, \omega, \mathcal{C}}$.

We write

$$
\phi^{N}(x)=\phi^{N, \omega, \mathcal{C}}(x)=E^{N, x}\left(I^{N}(1)\right)
$$

where $E^{N, x}=E^{N, x, \omega, \mathcal{C}}$ is the law of the chain $\xi^{N}$, with $\xi^{N}(0)=x \in \mathcal{C}$. By (5.6), the diffusion constant $D^{N}$ is given by

$$
D^{N}=\left\langle I^{N}(1)^{2}\right\rangle_{\alpha^{N}}-2\left\langle\phi^{N}\left(1-Q^{N}\right)^{-1} \phi^{N}\right\rangle_{\alpha^{N}}
$$


Using (5.7), we remark that $D^{N}$ is also given as the a.s. limit of the sample variance,

$$
D_{N}=\lim _{n \rightarrow \infty} \frac{1}{n}\left(\sum_{k=1}^{n} F^{N}\left(\xi^{N}(k-1), \xi^{N}(k)\right)\right)^{2} \text { a.s. }
$$

Noting that

$$
\sum_{k=1}^{n} F^{N}\left(\xi^{N}(k-1), \xi^{N}(k)\right)=\xi_{1}^{N}(n)-\xi_{1}^{N}(0)+2 N \sum_{k=1}^{n} G^{N}\left(\xi^{N}(k-1), \xi^{N}(k)\right)
$$

and $\left|\xi_{1}^{N}(n)-\xi_{1}^{N}(0)\right| \leq 2 N$, we deduce that

$$
D^{N}=4 N^{2} \lim _{n \rightarrow \infty} \frac{1}{n}\left(\sum_{k=1}^{n} G^{N}\left(\xi^{N}(k-1), \xi^{N}(k)\right)\right)^{2} \quad \text { a.s. }
$$

\subsection{Computing the Diffusion Constants}

It was proved in Theorem 4.1 that, with probability tending to 1 as $N \rightarrow \infty$, there exists a unique equivalence class which straddles $B^{N}$. It is clear from (6.16) that, on $\Omega^{N}$, we have that $D^{N}=D^{N, \omega, \mathcal{D}}=0$ for equivalence classes $\mathcal{D}$ which do not straddle $B^{N}$.

Lemma 6.2. Assume that $\omega$ satisfies (6.4) and that $\mathcal{C}$ straddles $B^{N}$. Then

$$
D^{N}=\frac{4 N^{2} \sigma^{N}}{d|\mathcal{C}|\left[1+\sigma^{N} / n^{N}(r, l)\right]}
$$

where $\sigma^{N}=\sigma^{N, \omega, \mathcal{C}}$ is the electrical conductance introduced below (4.5).

Proof. Assume that $\mathcal{C}$ straddles $B^{N}$. With

$$
\gamma^{N}(x)=E^{N, x}\left[G^{N}\left(\xi^{N}(0), \xi^{N}(1)\right)\right],
$$

we have that

$$
\gamma^{N}(x)= \begin{cases}0 & \text { if } x \in \mathcal{M} \\ Q^{N}(r, l) & \text { if } x=r \\ -Q^{N}(l, r) & \text { if } x=l\end{cases}
$$

By (6.15)-(6.16) and Theorem 5.1,

$$
(2 N)^{-2} D^{N}=\left\langle G^{N}\left(\xi^{N}(0), \xi^{N}(1)\right)^{2}\right\rangle_{\alpha^{N}}-2\left\langle\gamma^{N}\left(1-Q^{N}\right)^{-1} \gamma^{N}\right\rangle_{\alpha^{N}} .
$$

The first term here is given by

$$
\left\langle G^{N}\left(\xi^{N}(0), \xi^{N}(1)\right)^{2}\right\rangle_{\alpha^{N}}=\frac{n^{N}(r, l)}{d|\mathcal{C}|}
$$

In order to study the last term in (6.19), we look for solutions $\chi$ to the equation

$$
\left(1-Q^{N}\right) \chi=\gamma^{N}
$$


In particular, $\left[\left(1-Q^{N}\right) \chi\right](v)=0$ for $v \in \mathcal{M}$; that is, $\chi$ is harmonic on $\mathcal{M}$.

It turns out that such $\chi$ may be represented as the potential function of a certain electrical network, namely the network introduced in Section 4. As we did there, we construct a network having node set $\mathcal{V}$ by placing exactly $\kappa^{N}(v, w)=n^{\omega^{N}}(v,[w])$ unit resistors in parallel between each pair $v, w \in \mathcal{V}$ satisfying $\{v, w\} \neq\{r, l\}$; we allow the case $v=w$. No direct connection is made between $r$ and $l$.

Let $V$ be the potential function induced in this network when a unit potential difference between $r$ and $l$ is established. That is, $\left[\left(1-Q^{N}\right) V\right](v)=0$ for all $v \in \mathcal{M}$, and we shall take $V(r)=1, V(l)=0$. We write $\sigma^{N}=\sigma^{N, \omega, \mathcal{C}}$ for the conductance of the network between $r$ and $l$.

It follows from (6.18), (6.21), and the theory of electrical networks, that any solution $\chi$ of (6.21) may be expressed in the form $\chi=a V+b$ for some constants $a, b$ whose values are to be determined. We prove next that $b$ is arbitrary, and that

$$
a=\frac{n^{N}(r, l)}{n^{N}(r, l)+\sigma^{N}} .
$$

It suffices to prove that $a V+b$ satisfies (6.21) when (6.22) holds. Certainly $a V+b$ satisfies (6.21) at all nodes $v \in \mathcal{M}$, since $V$ is harmonic on $\mathcal{M}$ and $b$ is a constant.

We have that

$$
\begin{aligned}
{\left[\left(1-Q^{N}\right) V\right](r) } & =\sum_{v \in \mathcal{V}}\left(\delta_{r, v}-Q^{N}(r, v)\right) V(v) \\
& =Q^{N}(r, l)+\sum_{v \in \mathcal{M}} Q^{N}(r, v)(1-V(v)),
\end{aligned}
$$

and

$$
\left[\left(1-Q^{N}\right) V\right](l)=-Q^{N}(l, r)-\sum_{v \in \mathcal{M}} Q^{N}(l, v) V(v) .
$$

The net current in the network at $r$ equals the net current at $l$, and both are equal to the effective conductance $\sigma^{N}$ of the network. The current flowing along a light path is equal to the potential difference between its endpoints. Therefore the net current at $r$ equals the sum over all paths ending in $r$ of the potential differences between their endpoints, which equals

$$
\sum_{v \in \mathcal{M}} n^{N}(r, v)(1-V(v))=2 d|r| \sum_{v \in \mathcal{M}} Q^{N}(r, v)(1-V(v))
$$

and similarly the net current at $l$ equals

$$
\sum_{v \in \mathcal{M}} n^{N}(v, l) V(v)=2 d|l| \sum_{v \in \mathcal{M}} Q^{N}(l, v) V(v) .
$$

Therefore

$$
|r| \sum_{v \in \mathcal{M}} Q^{N}(r, v)(1-V(v))=|l| \sum_{v \in \mathcal{M}} Q^{N}(l, v) V(v)=\frac{\sigma^{N}}{2 d} .
$$

Since

$$
|r| Q^{N}(r, l)=|l| Q^{N}(l, r)=\frac{n^{N}(r, l)}{2 d},
$$


we have by (6.23)-(6.24) that

$$
|r|\left[\left(1-Q^{N}\right) V\right](r)=-|l|\left[\left(1-Q^{N}\right) V\right](l)=\frac{1}{2 d}\left[n^{N}(r, l)+\sigma^{N}\right] .
$$

Hence $a V+b$ satisfies (6.21) if and only if $a$ is given by (6.22). We choose $a$ accordingly.

Returning to the last term of (6.19), we have now that

$$
2\left\langle\gamma^{N}\left(1-Q^{N}\right)^{-1} \gamma^{N}\right\rangle_{\alpha^{N}}=2\left\langle\gamma^{N}(a V+b)\right\rangle_{\alpha^{N}}=\frac{a n^{N}(r, l)}{d|\mathcal{C}|} .
$$

Combining this with (6.19) and (6.20), we deduce that

$$
(2 N)^{-2} D^{N}=(1-a) \frac{n^{N}(r, l)}{d|\mathcal{C}|}
$$

whence (6.17) follows from (6.22).

\subsection{Comparison of $D$ with the $D^{N, \omega, \mathcal{C}}$}

Let $\mathbb{P}^{N}$ be the measure $\mathbb{P}$ conditioned on the event $\Omega^{N}$ (see the discussion above (6.4)), and assume that $\omega \in \Omega^{N}$. If $\mathcal{C}$ is an equivalence class of rw points of $B^{N}$ under the relation $\leftrightarrow_{N}$ (introduced above (4.3)), let $\mathcal{V}^{N, \omega, \mathcal{C}}$ be as in (6.5) and $\alpha^{N, \omega, \mathcal{C}}$ as in (6.13). Let

$$
\mathcal{V}^{N, \omega}=\bigcup_{\mathcal{C}} \mathcal{V}^{N, \omega, \mathcal{C}}
$$

where the union is over all such equivalence classes $\mathcal{C}$, and define a measure $\alpha^{N, \omega}$ on $\mathcal{V}^{N, \omega}$ by

$$
\alpha^{N, \omega}(x)=\frac{|\mathcal{C}|}{\left|\mathcal{W}^{N}(\omega)\right|} \alpha^{N, \omega, \mathcal{C}}(x) \quad \text { whenever } x \in \mathcal{V}^{N, \omega, \mathcal{C}} .
$$

We define the function $\phi^{N, \omega}$ on $\mathcal{V}^{N, \omega}$ by

$$
\phi^{N, \omega}(x)=\phi^{N, \omega, \mathcal{C}}(x) \quad \text { whenever } x \in \mathcal{V}^{N, \omega, \mathcal{C}}
$$

where $\phi^{N, \omega, \mathcal{C}}$ is given in (6.14). Let $\left(\xi^{N, \omega, \mathcal{C}}(n): n \geq 0\right)$ be as in (6.9), and define the process $\left(\xi^{N, \omega}(n): n \geq 0\right)$ on $\mathcal{V}^{N, \omega}$ by letting $\xi^{N, \omega}(n)=\xi^{N, \omega, \mathcal{C}}(n)$ if $\xi^{N, \omega}(0) \in \mathcal{V}^{N, \omega, \mathcal{C}}$. Next, let

$$
i^{N, \omega}(1)=F^{N, \omega, \mathcal{C}}\left(x, \xi^{N, \omega}(1)\right) \quad \text { whenever } \xi^{N, \omega}(0)=x \in \mathcal{V}^{N, \omega, \mathcal{C}},
$$

where $F^{N, \omega, \mathcal{C}}$ is as in (6.12). Finally, for $x \in \mathcal{V}^{N, \omega}$, let $Q^{N, \omega}(x, \cdot)$ be the transition probability distribution on $\mathcal{V}^{N, \omega}$ defined by

$$
Q^{N, \omega}(x, d y)=Q^{N, \omega, \mathcal{C}}(x, d y) \quad \text { whenever } x \in \mathcal{V}^{N, \omega, \mathcal{C}},
$$

where $Q^{N, \omega, \mathcal{C}}$ is given by (6.7). Finally, let

$$
D^{N, \omega}=\left\langle i^{N, \omega}(1)^{2}\right\rangle_{\alpha^{N, \omega}}-2\left\langle\phi^{N, \omega}\left(1-Q^{N, \omega}\right)^{-1} \phi^{N, \omega}\right\rangle_{\alpha^{N, \omega}} .
$$

The next lemma is closely related to Proposition 5.13 of [11]. 
Lemma 6.3. Let $p_{\text {rw }}>0$, let $\Psi=\mathbb{P}^{*}\left(\Omega^{* *}\right)$, and let $D^{*}$ be the analogue of $D$ with $\mathbb{P}^{* *}$ replaced by $\mathbb{P}^{*}$ in (5.10). Let $A=A\left(p_{\mathrm{rw}}\right)>0$ be given as in Theorem 3.3, and suppose that either $1-p_{\mathrm{rw}}-p_{+}<A$ or $p_{\mathrm{rw}}>p_{\mathrm{c}}$.

(i) We have that $\Psi>0$ and $D^{*}=\Psi D$.

(ii) For a fixed integer $k \geq 0$,

$$
\lim _{N \rightarrow \infty} \mathbb{P}^{N}\left(\left\langle\phi^{N, \omega}\left(Q^{N, \omega}\right)^{k} \phi^{N, \omega}\right\rangle_{\alpha^{N, \omega}}\right)=\left\langle\phi Q^{k} \phi\right\rangle_{\mathbb{P}^{*}},
$$

where $\phi=\Phi_{1}$ as in (5.10).

(iii) We have that $D^{*} \geq \lim \sup _{N \rightarrow \infty} \mathbb{P}^{N}\left(D^{N, \omega}\right)$.

Proof. The strict positivity of $\Psi$ is given in Theorem 3.3. The remainder of part (i) follows as for the proof of (4.26a) of [11, p. 825]. (See the proof of (4.37) on page 827 of [11].) The argument given there uses the fact, implied by Theorem 3.3, that the radius of a finite equivalence class containing the origin has a finite second moment under $\mathbb{P}^{*}$.

Next we prove (ii). Fix $k \geq 0$. We define

$$
J^{N}=\left\{x \in B^{N}:\|x\| \leq N-(k+2) N^{\rho}\right\} .
$$

Then, for some constant $C$ depending on $k,\left|B^{N} \backslash J^{N}\right| \leq C N^{d-1+\rho}$, since $\rho<1$. It follows that

$$
\left\langle\phi Q^{k} \phi\right\rangle_{\mathbb{P}^{*}}=\lim _{N \rightarrow \infty}\left\{\frac{\left|J^{N}\right|}{\left|B^{N}\right|}\left\langle\phi Q^{k} \phi\right\rangle_{\mathbb{P}^{*}}\right\} .
$$

We turn now to the left side of (6.27). By the definition of $\alpha^{N, \omega}$,

$$
\left\langle\phi^{N, \omega}\left(Q^{N, \omega}\right)^{k} \phi^{N, \omega}\right\rangle_{\alpha^{N, \omega}}=\frac{1}{\left|\mathcal{W}^{N}(\omega)\right|} \sum_{x \in \mathcal{V}^{N, \omega}}|x| \phi^{N, \omega}(x)\left[\left(Q^{N, \omega}\right)^{k} \phi^{N, \omega}\right](x) .
$$

We split the last sum into two parts depending on whether or not $x \in B^{N} \backslash J^{N}$. Since $\phi^{N, \omega}$ is uniformly bounded by $N^{\rho}$ under $\mathbb{P}^{N}$, the first sum (over $x \in B^{N} \backslash J^{N}$ and composite $x$ ) is bounded above by

$$
N^{2 \rho} \frac{\left|\mathcal{W}(\omega) \cap\left(B^{N} \backslash J^{N}\right)\right|}{\left|\mathcal{W}^{N}(\omega)\right|} \leq C_{1} N^{2 \rho} \frac{\left|B^{N} \backslash J^{N}\right|}{N^{(1-\rho) d}} \leq C_{2} N^{\rho(d+3)-1} \quad \mathbb{P}^{N} \text {-a.s. }
$$

for appropriate constants $C_{i}$ (we have used the fact that, $\mathbb{P}^{N}$-a.s., every sphere of radius $N^{\rho}$ contains some rw point). This tends to 0 as $N \rightarrow \infty$, since $\rho<(d+3)^{-1}$; cf. (6.1).

Therefore,

$$
\begin{aligned}
\mathbb{P}^{N}\left(\left\langle\phi^{N, \omega}\left(Q^{N, \omega}\right)^{k} \phi^{N, \omega}\right\rangle_{\alpha^{N, \omega}}\right) & \\
& =\mathrm{o}(1)+p_{\mathrm{rw}} \sum_{x \in J^{N}} \mathbb{P}_{x}^{N}\left(\frac{1}{\left|\mathcal{W}^{N}(\omega)\right|} \phi^{N, \omega}(x)\left[\left(Q^{N, \omega}\right)^{k} \phi^{N, \omega}\right](x)\right)
\end{aligned}
$$

where $\mathbb{P}_{x}^{N}$ denotes $\mathbb{P}^{N}$ conditioned on $x$ being a rw point.

Let $X^{N, \omega}$ be the Markov chain having state space $\mathcal{V}^{N, \omega}+2 N \mathbb{Z} u_{1}$ and transition probability measure $q^{N, \omega}(x, \cdot)=q^{N, \omega, \mathcal{C}}(x, \cdot)$ whenever $x \in \mathcal{V}^{N, \omega, \mathcal{C}}+2 N \mathbb{Z} u_{1}$ (see (6.8)). For given $\omega \in \Omega^{N}$ and $x \in \mathcal{W}^{N}(\omega)$, the processes $X^{\omega}$ and $X^{N, \omega}$ may be coupled in such a way that

$$
X^{\omega}(0)=X^{N, \omega}(0)=x, \quad X^{\omega}(n)=X^{N, \omega}(n) \quad \text { for } n<\theta^{N, x, \omega},
$$


where

$$
\theta^{N, x, \omega}=\min \left\{m \geq 0: X^{\omega}(m) \in \partial \mathcal{W}^{N}(\omega)\right\}
$$

is the first hitting time by $X^{\omega}$ of the set

$$
\partial \mathcal{W}^{N}(\omega)=\left\{x \in \mathcal{W}^{N}: \exists y \notin B^{N} \text { with } x \leftrightarrow \omega^{\omega^{N}} y\right\} ;
$$

note that $\theta^{N, x, \omega} \geq k+1$ for $x \in J^{N}$, by (6.28). Hence, using (6.32),

$$
\mathbb{P}^{N}\left(\left\langle\phi^{N, \omega}\left(Q^{N, \omega}\right)^{k} \phi^{N, \omega}\right\rangle_{\alpha^{N, \omega}}\right)=\mathrm{o}(1)+p_{\mathrm{rw}} \sum_{x \in J^{N}}\left\langle\frac{1}{\left|\mathcal{W}^{N}(\omega)\right|} \psi_{x}(\omega)\right\rangle_{\mathbb{P}_{x}^{N}}
$$

where $\psi_{x}(\omega)=\phi\left(\tau_{-x} \omega\right)\left[Q^{k} \phi\right]\left(\tau_{-x} \omega\right)$. Replacing $\mathbb{P}_{x}^{N}$ by $\mathbb{P}_{x}$ (i.e., $\mathbb{P}$ conditioned on $x$ being a rw point), we have that

$$
\begin{aligned}
& \mathbb{P}^{N}\left(\left\langle\phi^{N, \omega}\left(Q^{N, \omega}\right)^{k} \phi^{N, \omega}\right\rangle_{\alpha^{N, \omega}}\right) \\
& \quad=\mathrm{o}(1)+\frac{1}{\left|B^{N}\right| \mathbb{P}^{*}\left(\Omega^{N}\right)} \sum_{x \in J^{N}}\left\langle\psi_{x}\right\rangle_{\mathbb{P}_{x}}+R_{N}(1)-R_{N}(2) \\
& \quad=\mathrm{o}(1)+\frac{\left|J^{N}\right|}{\left|B^{N}\right| \mathbb{P}^{*}\left(\Omega^{N}\right)}\left\langle\psi_{0}\right\rangle_{\mathbb{P}^{*}}+R_{N}(1)-R_{N}(2)
\end{aligned}
$$

where

$$
\begin{aligned}
& R_{N}(1)=\frac{1}{\left|B^{N}\right| \mathbb{P}^{*}\left(\Omega^{N}\right)} \sum_{x \in J^{N}}\left\langle\left(\frac{p_{\mathrm{rw}}\left|B^{N}\right|}{\left|\mathcal{W}^{N}(\omega)\right|}-1\right) \psi_{x}(\omega)\right\rangle_{\mathbb{P}_{x}} \\
& R_{N}(2)=\frac{1}{\left|B^{N}\right| \mathbb{P}^{*}\left(\Omega^{N}\right)} \sum_{x \in J^{N}}\left\langle\frac{p_{\mathrm{rw}}\left|B^{N}\right|}{\left|\mathcal{W}^{N}(\omega)\right|}\left(1-1_{\left\{\Omega^{N}\right\}}\right) \psi_{x}(\omega)\right\rangle_{\mathbb{P}_{x}} .
\end{aligned}
$$

Now $\mathbb{P}^{*}\left(\Omega^{N}\right) \rightarrow 1$ as $N \rightarrow \infty$, whence, by (6.29),

$$
\frac{\left|J^{N}\right|}{\left|B^{N}\right| \mathbb{P}^{*}\left(\Omega^{N}\right)}\left\langle\psi_{0}\right\rangle_{\mathbb{P}^{*}} \rightarrow\left\langle\phi Q^{k} \phi\right\rangle_{\mathbb{P}^{*}} \quad \text { as } N \rightarrow \infty .
$$

Also, by the Cauchy-Schwarz inequality and properties of the binomial distribution,

$$
\left|R_{N}(1)\right| \leq \frac{1}{\left|B^{N}\right| \mathbb{P}^{*}\left(\Omega^{N}\right)} \sum_{x \in J^{N}}\left\{\mathbb{P}_{x}\left(\left[\frac{p_{\mathrm{rw}}\left|B^{N}\right|}{\left|\mathcal{W}^{N}\right|}-1\right]^{2}\right) \mathbb{P}_{x}\left(\psi_{x}^{2}\right)\right\}^{1 / 2} \rightarrow 0
$$

as $N \rightarrow \infty$. (Remember that $\mathbb{P}_{x}\left(\psi_{x}^{2}\right)=\mathbb{P}_{0}\left(\psi_{0}^{2}\right)<\infty$ by Theorem 3.2.) Secondly,

$$
\left|R_{N}(2)\right| \leq \frac{1}{\left|B^{N}\right| \mathbb{P}^{*}\left(\Omega^{N}\right)} \sum_{x \in J^{N}}\left\{\mathbb{P}_{x}\left(\overline{\Omega^{N}}\right) \mathbb{P}_{x}\left(\left[\frac{p_{\mathrm{rw}}\left|B^{N}\right|}{\left|\mathcal{W}^{N}\right|} \psi_{x}\right]^{2}\right)\right\}^{1 / 2} .
$$

Now

$$
\mathbb{P}_{x}\left(\overline{\Omega^{N}}\right) \leq \frac{\mathbb{P}\left(\overline{\Omega^{N}}\right)}{p_{\mathrm{rw}}} \rightarrow 0 \quad \text { as } N \rightarrow \infty .
$$


We apply the Cauchy-Schwarz inequality once again, and use properties of the binomial distribution together with the fact that $\mathbb{P}_{x}\left(\psi_{x}^{4}\right)=\mathbb{P}_{0}\left(\psi_{0}^{4}\right)<\infty$, obtaining thereby that $\left|R_{N}(2)\right| \rightarrow 0$ as $N \rightarrow \infty$. The required equation (6.27) follows from (6.29) and (6.33)(6.34).

Finally, we prove (iii). There is an argument using Fatou's lemma which we seek to apply. Unfortunately a minor difficulty arises, namely that $\left\langle\phi Q^{k} \phi\right\rangle_{\mathbb{P}^{*}}$ can be negative as well as positive; certainly $\left\langle\phi Q^{k} \phi\right\rangle_{\mathbb{P}^{*}} \geq 0$ for even values of $k$, since $\mathbb{P}^{*}$ is stationary and reversible for $\xi$, but the reverse inequality can hold when $k$ is odd. In order to obviate this annoying detail, we move from discrete to continuous time. Let $M=(M(t): t \geq 0)$ be a Poisson process having intensity 1 and right-continuous sample paths, independent of all random variables discussed so far in this paper. For any sequence $Z=(Z(n): n \geq 0)$, of random variables we define the corresponding continuous-time process $\left(Z_{t}: t \geq 0\right)$, by $Z_{t}=Z(M(t))$.

Before continuing, we note one elementary property of the 'Poissonised' process.

Lemma 6.4. Let $Z$ be a random sequence satisfying $n^{-1} E\left(Z(n)^{2}\right) \rightarrow \sigma^{2}$ as $n \rightarrow \infty$. If $M$ is independent of the $Z(n)$, then $t^{-1} E\left(Z_{t}^{2}\right) \rightarrow \sigma^{2}$ as $t \rightarrow \infty$.

Proof. This follows in an elementary way from the fact that

$$
E\left(Z_{t}^{2}\right)=\sum_{n=0}^{\infty} E\left(Z(n)^{2}\right) P(M(t)=n) .
$$

Central limit theorems are valid for Poissonised versions of the processes considered above. As shown in $[11,24]$, there is a version of Theorem 5.1 for continuous time. We do not present the full details of this, since they are very close to those presented already. However, we shall make use of the following consequences.

Consider the two continuous-time processes $X_{1, t}^{\omega}$ and $X_{1, t}^{N, \omega}$; these are the first-coordinate processes of $X_{t}^{\omega}$ and $X_{t}^{N, \omega}$. These processes have zero means, which is to say that

$$
\mathbb{P}^{*}\left(P_{0}^{\omega}\left(X_{1, t}^{\omega}\right)\right)=\left\langle X_{1, t}^{N, \omega}\right\rangle_{\alpha^{N, \omega}}=0
$$

and their second moments satisfy

$$
\begin{aligned}
\frac{1}{t} \mathbb{P}^{*}\left(P_{0}^{\omega}\left[\left(X_{1, t}^{\omega}\right)^{2}\right]\right) & \rightarrow \Delta^{*}, \\
\frac{1}{t}\left\langle\left(X_{1, t}^{N, \omega}\right)^{2}\right\rangle_{\alpha^{N, \omega}} & \rightarrow \Delta^{N, \omega},
\end{aligned}
$$

as $t \rightarrow \infty$, for some constant $\Delta^{*}$ and random variable $\Delta^{N, \omega}$. Furthermore, by $[11$, Thm 2.2], $\Delta^{*}$ and $\Delta^{N, \omega}$ may be represented as

$$
\begin{aligned}
\Delta^{*} & =\left\langle i(1)^{2}\right\rangle_{\mathbb{P}^{*}}+2\left\langle\phi, L^{-1} \phi\right\rangle_{\mathbb{P}^{*}} \\
& =\left\langle i(1)^{2}\right\rangle_{\mathbb{P}^{*}}-2 \int_{0}^{\infty}\left\langle\phi, Q_{t} \phi\right\rangle_{\mathbb{P}^{*}} d t, \\
\Delta^{N, \omega} & =\left\langle I^{N}(1)^{2}\right\rangle_{\alpha^{N, \omega}}+2\left\langle\phi^{N, \omega},\left(L^{N}\right)^{-1} \phi^{N, \omega}\right\rangle_{\alpha^{N, \omega}} \\
& =\left\langle I^{N}(1)^{2}\right\rangle_{\alpha^{N, \omega}}-2 \int_{0}^{\infty}\left\langle\phi^{N, \omega}, Q_{t}^{N} \phi^{N, \omega}\right\rangle_{\alpha^{N, \omega}} d t,
\end{aligned}
$$

where $L$ and $Q_{t}$ (resp. $L^{N}=L^{N, \omega}$ and $Q_{t}^{N}=Q_{t}^{N, \omega}$ ) are the generator and transition semigroup of $X_{t}^{\omega}$ (resp. $\left.X_{t}^{N, \omega}\right)$; cf. (5.10) and (6.15).

Parts $(\mathrm{a}, \mathrm{c})$ of the following lemma imply Lemma 6.3(iii). 
Lemma 6.5. Let $p_{\mathrm{rw}}>0$, and let $A=A\left(p_{\mathrm{rw}}\right)>0$ be given as in Lemma 6.3. Suppose that either $1-p_{\mathrm{rw}}-p_{+}<A$ or $p_{\mathrm{rw}}>p_{\mathrm{c}}$.

(a) We have that $\Delta^{*}=D^{*}$ and $\Delta^{N, \omega}=D^{N, \omega}$.

(b) For a fixed time $t \geq 0$,

$$
\left\langle\phi^{N, \omega}, Q_{t}^{N} \phi^{N, \omega}\right\rangle_{\alpha^{N, \omega}} \geq 0, \quad \lim _{N \rightarrow \infty} \mathbb{P}^{N}\left(\left\langle\phi^{N, \omega}, Q_{t}^{N} \phi^{N, \omega}\right\rangle_{\alpha^{N, \omega}}\right)=\left\langle\phi, Q_{t} \phi\right\rangle_{\mathbb{P}^{*}} .
$$

(c) We have that $\Delta^{*} \geq \lim \sup _{N \rightarrow \infty} \mathbb{P}^{N}\left(\Delta^{N, \omega}\right)$.

Proof. Part (a) is a consequence of Lemma 6.4. Turning to (b), we have by the fact that $X_{t}^{N, \omega}$ is reversible with respect to $\alpha^{N, \omega}$ that

$$
\left\langle\phi^{N, \omega}, Q_{2 t}^{N} \phi^{N, \omega}\right\rangle_{\alpha^{N, \omega}}=\left\langle Q_{t}^{N} \phi^{N, \omega}, Q_{t}^{N} \phi^{N, \omega}\right\rangle_{\alpha^{N, \omega}} \geq 0
$$

for any $t \geq 0$. The second part of (6.39) follows thus from (6.27). Note first that, under the conditions of the lemma, there exists a constant $C$ such that

$$
\mathbb{P}^{N}\left(\left\langle\left(\phi^{N, \omega}\right)^{2}\right\rangle_{\alpha^{N, \omega}}\right) \leq C, \quad\left\langle\phi^{2}\right\rangle_{\mathbb{P}^{*}} \leq C .
$$

[Such inequalities may be obtained from (4.6) and Theorem 3.2 respectively.] Also,

$$
\begin{aligned}
\left\langle\phi^{N, \omega}, Q_{t}^{N} \phi^{N, \omega}\right\rangle_{\alpha^{N, \omega}} & =\sum_{k=0}^{\infty}\left\langle\phi^{N, \omega},\left(Q^{N, \omega}\right)^{k} \phi^{N, \omega}\right\rangle_{\alpha^{N, \omega}} P(M(t)=k) \\
\left\langle\phi, Q_{t} \phi\right\rangle_{\mathbb{P}^{*}} & =\sum_{k=0}^{\infty}\left\langle\phi, Q^{k} \phi\right\rangle_{\mathbb{P}^{*}} P(M(t)=k)
\end{aligned}
$$

whence, for $K \geq 1$,

$$
\begin{gathered}
\left|\mathbb{P}^{N}\left(\left\langle\phi^{N, \omega}, Q_{t}^{N} \phi^{N, \omega}\right\rangle_{\alpha^{N, \omega}}\right)-\left\langle\phi, Q_{t} \phi\right\rangle_{\mathbb{P}^{*}}\right| \\
\leq \sum_{k=0}^{K}\left|\left\langle\phi^{N, \omega},\left(Q^{N, \omega}\right)^{k} \phi^{N, \omega}\right\rangle_{\alpha^{N, \omega}}-\left\langle\phi, Q^{k} \phi\right\rangle_{\mathbb{P}^{*}}\right| P(M(t)=k) \\
\quad+\left\{\mathbb{P}^{N}\left(\left\langle\left(\phi^{N, \omega}\right)^{2}\right\rangle_{\alpha^{N, \omega}}\right)+\left\langle\phi^{2}\right\rangle_{\mathbb{P}^{*}}\right\} P(M(t)>K) .
\end{gathered}
$$

Therefore, by (6.27),

$$
\limsup _{N \rightarrow \infty}\left|\mathbb{P}^{N}\left(\left\langle\phi^{N, \omega}, Q_{t}^{N} \phi^{N, \omega}\right\rangle_{\alpha^{N, \omega}}\right)-\left\langle\phi, Q_{t} \phi\right\rangle_{\mathbb{P}^{*}}\right| \leq 2 C P(M(t)>K),
$$

which tends to 0 as $K \rightarrow \infty$. This proves part (b).

Finally we prove (c). By (6.39) and Fatou's lemma,

$$
\begin{aligned}
\left\langle\phi, L^{-1} \phi\right\rangle_{\mathbb{P}^{*}} & =-\int_{0}^{\infty}\left\langle\phi, Q_{t} \phi\right\rangle_{\mathbb{P}^{*}} d t \\
& =-\int_{0}^{\infty}\left\{\liminf _{N \rightarrow \infty} \mathbb{P}^{N}\left(\left\langle\phi^{N, \omega}, Q_{t}^{N} \phi^{N, \omega}\right\rangle_{\alpha^{N, \omega}}\right)\right\} d t \\
& \geq-\liminf _{N \rightarrow \infty} \int_{0}^{\infty} \mathbb{P}^{N}\left(\left\langle\phi^{N, \omega}, Q_{t}^{N} \phi^{N, \omega}\right\rangle_{\alpha^{N, \omega}}\right) d t \\
& =\limsup _{N \rightarrow \infty} \mathbb{P}^{N}\left(\left\langle\phi^{N, \omega},\left(L^{N}\right)^{-1} \phi^{N, \omega}\right\rangle_{\alpha^{N, \omega}}\right),
\end{aligned}
$$


where we have used Fubini's theorem at the last step. Moreover,

$$
\left\langle I^{N}(1)^{2}\right\rangle_{\alpha^{N, \omega}}=\frac{1}{\left|\mathcal{W}^{N}(\omega)\right|} \sum_{x \in \mathcal{V}^{N, \omega}}|x| E^{N, x}\left[\left(X_{1}^{N, \omega}(1)-X_{1}^{N, \omega}(0)\right)^{2}\right],
$$

where $E^{N, x}$ is the law of the random walk on $\mathcal{V}^{N, \omega}$ starting at the (possibly composite) state $x$; cf. (6.15). Arguing as in the proof of (6.27), we obtain that

$$
\begin{aligned}
\lim _{N \rightarrow \infty} \mathbb{P}^{N}\left(\left\langle I^{N}(1)^{2}\right\rangle_{\alpha^{N, \omega}}\right) & =\lim _{N \rightarrow \infty}\left\{\frac{1}{\left|B^{N}\right|} \sum_{x \in J^{N}}\left\langle E_{x}^{\omega}\left[\left(X_{1}^{\omega}(1)-X_{1}^{\omega}(0)\right)^{2}\right]\right\rangle_{\mathbb{P}_{x}}\right\} \\
& =\left\langle E_{0}^{\omega}\left[\left(X_{1}^{\omega}(1)-X_{1}^{\omega}(0)\right)^{2}\right]\right\rangle_{\mathbb{P}^{*}}=\left\langle i(1)^{2}\right\rangle_{\mathbb{P}^{*}},
\end{aligned}
$$

where $J^{N}$ is given by (6.28) with $k=2$, say. We obtain part (c) by combining (6.37)-(6.38) and $(6.41)-(6.42)$.

\subsection{Conclusion}

Finally, we deduce that $D>0$, as claimed in Lemma 6.1. Let $p_{\text {rw }}>0$ and let $A$ be chosen as in Theorem 4.1. We note from (3.6) that this value of $A$ may be taken from Theorem 3.3. Assume that either $1-p_{\text {rw }}-p_{+}<A$ or $p_{\text {rw }}>p_{\mathrm{c}}$. When statements (a) $-(\mathrm{e})$ of Theorem 4.1 hold, we have by Lemma 6.2 (together with the remark preceding it) and (6.26) that

$$
D^{N, \omega} \geq\left(\frac{4 N^{2}\left(c_{3} N^{d-2}\right)}{d|\mathcal{N}|\left[1+c_{3} N^{d-2}\left(c_{2} N^{d-1}\right)^{-1}\right]}\right) \frac{|\mathcal{N}|}{(2 N+1)^{d}} \geq c_{4}>0,
$$

where $\mathcal{N}$ is the largest equivalence class of $\mathcal{W}^{N}$ under $\leftrightarrow_{N}$. Therefore $\mathbb{P}\left(D^{N, \omega}>c_{4}\right) \rightarrow 1$ as $N \rightarrow \infty$, whence

$$
\liminf _{N \rightarrow \infty} \mathbb{P}^{N}\left(D^{N, \omega}\right) \geq c_{4}>0
$$

implying by Lemma $6.3\left(\mathrm{i}\right.$, iii) that $D=\theta D^{*}>0$.

\section{Proof of Theorem 2.2}

We shall deduce Theorem 2.2 from Theorem 2.1 with the aid of the ergodic theorem. In order to achieve this, we shall adapt various standard arguments used to prove functional central limit theorems. These arguments may be found in $[4,12,15]$.

Let $\omega \in \Omega^{* *}$ and $u \in I^{ \pm}=\left\{ \pm u_{i}: 1=1,2, \ldots, d\right\}$. We define $l(\omega, u)$ to be the number of edges in the light path starting at the origin 0 , heading off in the direction $u$, and ending at the first rw point encountered subsequently. Then

$$
m=\mathbb{P}^{* *}\left(\frac{1}{2 d} \sum_{u \in I^{ \pm}} l(\omega, u)\right)=\mathbb{P}^{* *}\left(l\left(\omega, u_{1}\right)\right) .
$$

Cf. (2.5). Let $W=\left(W_{t}: t \geq 0\right)$ be a standard Brownian motion in $\mathbb{R}^{d}$, let $D$ be as in Theorem 2.1, and write $V_{t}=\sqrt{D} W_{t / m}$. We shall prove that $Y^{\varepsilon, \cdot}$ converges $\mathbb{P}^{* *}$-dp to the process $V$.

We begin by stating some general facts. Let $(S, \rho)$ be a complete separable metric space, and $\mathcal{B}(S)$ the set of its Borel subsets. Let $\mathcal{P}(S)$ be the collection of probability measures on $(S, \mathcal{B}(S))$, and topologise $\mathcal{P}(S)$ by the topology of weak convergence. (See [4], pp. 236-239.) Let $\mathcal{B}(\mathcal{P}(S))$ be the corresponding Borel subsets of $\mathcal{P}(S)$. Let $\left(\Omega, \mathcal{F}, \mathbb{P}^{* *}\right)$ be a probability space and suppose that, for $\varepsilon>0$, the mapping $\omega \mapsto \nu_{\omega}^{\varepsilon}$ is a measurable function from $(\Omega, \mathcal{F})$ to $(\mathcal{P}(S), \mathcal{B}(\mathcal{P}(S)))$. 
Lemma 7.1. Let $\nu \in \mathcal{P}(S)$. The following statements are equivalent.

(a) $\int f d \nu_{\omega}^{\varepsilon} \rightarrow \int f d \nu$ in $\mathbb{P}^{* *}$-probability as $\varepsilon \rightarrow 0$, for all bounded continuous functions $f$ on $S$.

(b) $\int f d \nu_{\omega}^{\varepsilon} \rightarrow \int f d \nu$ in $\mathbb{P}^{* *}$-probability as $\varepsilon \rightarrow 0$, for all bounded uniformly continuous functions $f$ on $S$.

(c) $\pi\left(\nu_{\omega}^{\varepsilon}, \nu\right) \rightarrow 0$ in $\mathbb{P}^{* *}$-probability as $\varepsilon \rightarrow 0$, where $\pi$ is the Prohorov metric on $\mathcal{P}(S)$.

Proof. Suppose $T$ is any topological space, $\nu \in T$, and (for $\varepsilon>0$ ) $\nu^{\varepsilon}$ is a $T$-valued random variable on the probability space $\left(\Omega, \mathcal{F}, \mathbb{P}^{* *}\right)$. Let $\mathcal{N}_{\nu}$ be a sub-basis for the topology at $\nu$. Then $\nu^{\varepsilon} \rightarrow \nu$ in $\mathbb{P}^{* *}$-probability if and only if

$$
\mathbb{P}^{* *}\left(\nu^{\varepsilon} \in N\right) \rightarrow 1 \quad \text { as } \varepsilon \rightarrow 0 \text {, for every } N \in \mathcal{N}_{\nu} .
$$

By results in [4] (pp. 236-239, and Theorem 1.2 on p. 8), (a)-(c) may each be rephrased in the form (7.2) for a suitable choice of sub-basis $\mathcal{N}_{\nu}$ for the topology of weak convergence on $T=\mathcal{P}(S)$ at $\nu$.

If (a)-(c) hold, we say that $\nu_{\omega}^{\varepsilon}$ converges weakly in $\mathbb{P}^{* *}$-probability to $\nu$, written $\nu_{\omega}^{\varepsilon} \rightarrow \nu$ $\mathbb{P}^{* *}$-wp.

Now suppose that $\left(\Omega^{\prime}, \mathcal{F}^{\prime}, \mathbb{P}^{\prime}\right)$ is another probability space and that, for $\varepsilon>0$, the mapping $\left(\omega, \omega^{\prime}\right) \mapsto X_{\omega, \omega^{\prime}}^{\varepsilon}$ is a measurable function from the product space $\left(\Omega \times \Omega^{\prime}, \mathcal{F} \times \mathcal{F}^{\prime}\right)$ to $(S, \mathcal{B}(S))$. Let $\nu \in \mathcal{P}(S)$. We shall say that $X^{\varepsilon}$ 'converges in distribution in $\mathbb{P}^{* *}$-probability' (abbreviated to ' $\mathbb{P}^{* *}$-dp') to $\nu($ as $\varepsilon \rightarrow 0)$ if $\mathbb{P}^{\prime}\left(\left\{\omega^{\prime}: X_{\omega, \omega^{\prime}}^{\varepsilon} \in \cdot\right\}\right) \rightarrow \nu(\cdot) \mathbb{P}^{* *}$-wp. We note that it is a consequence of Fubini's theorem that the mapping $\omega \mapsto \mathbb{P}^{\prime}\left(X_{\omega, \cdot}^{\varepsilon} \in \cdot\right)$ is a measurable function from $(\Omega, \mathcal{F})$ to $(\mathcal{P}(S), \mathcal{B}(\mathcal{P}(S)))$.

The next lemma follows from the characterisation of weak convergence contained in Lemma 7.1(b) (cf. Corollary 3.2 of [15], p. 110).

Lemma 7.2. Let $\nu \in \mathcal{P}(S)$. Suppose that, for $\varepsilon>0$, both $X_{\omega, \omega^{\prime}}^{\varepsilon}$ and $Y_{\omega, \omega^{\prime}}^{\varepsilon}$ are measurable maps from $\left(\Omega \times \Omega^{\prime}, \mathcal{F} \times \mathcal{F}^{\prime}\right)$ to $(S, \mathcal{B}(S))$, and that

$$
\begin{aligned}
X^{\varepsilon} & \rightarrow \nu \quad \mathbb{P}^{* *} \text {-dp as } \varepsilon \rightarrow 0, \\
\mathbb{P}^{\prime}\left(\rho\left(X_{\omega, .}^{\varepsilon}, Y_{\omega, .}^{\varepsilon}\right)>\eta\right) & \rightarrow 0 \quad \text { in } \mathbb{P}^{* *} \text {-probability as } \varepsilon \rightarrow 0, \text { for all } \eta>0 .
\end{aligned}
$$

Then $Y^{\varepsilon} \rightarrow \nu \mathbb{P}^{* *}$-dp as $\varepsilon \rightarrow 0$.

We now return to the particular situation discussed in this paper. We claim first that since $X^{\varepsilon, \omega}$ converges $\mathbb{P}^{* *}$-dp to $\sqrt{D} W($ as $\varepsilon \rightarrow 0)$, the process $\left(X^{\varepsilon, \omega}(t / m): t \geq 0\right)$ converges in $\mathbb{P}^{* *}$-probability to $(\sqrt{D} W(t / m): t \geq 0)$. We next indicate why this holds. Let $f$ be a bounded continuous real-valued function on the Skorohod space $\Delta=D\left([0, \infty), \mathbb{R}^{d}\right)$. The function on $\Delta$ defined by $(X(t): t \geq 0) \mapsto f((X(t / m): t \geq 0))$ is also bounded and continuous.

Note also that if $T>0$ and $X, Y \in \Delta$, then

$$
\rho(X, Y)<e^{-T}+\sup _{0 \leq t \leq T}|X(t)-Y(t)|,
$$

where $\rho$ is the Skorohod metric on $\Delta$ (see [15], p. 117). Therefore, by Lemma 7.2, it suffices to show that, for fixed $\eta>0$ and $T>0$,

$$
P_{0}^{\omega}\left(\sup _{0 \leq t \leq T}\left|Y^{\varepsilon, \omega}(t)-X^{\varepsilon, \omega}(t / m)\right|>\eta\right) \rightarrow 0 \quad \text { in } \mathbb{P}^{* *} \text {-probability, as } \varepsilon \rightarrow 0 .
$$


To this end, let $\theta_{0}, \theta_{1}, \ldots$ be the successive times $n$ at which $Y^{\omega}(n) \in \mathcal{W}(\omega)$. We shall couple the processes $X^{\omega}$ and $Y^{\omega}$ together by setting $X^{\omega}(k)=Y^{\omega}\left(\theta_{k}\right)$. For $t \geq 0$, let $N(t)$ be the number of renewals of the sequence $\theta_{0}, \theta_{1}, \ldots$ up to time $t$, i.e., $N(t)=\sup \{k \geq$ $\left.0: \theta_{k} \leq t\right\}$; we define a process $\tilde{X}^{\omega}$ by $\tilde{X}^{\omega}(n)=X^{\omega}(N(n))$. For $\varepsilon>0$ and $t \geq 0$, define $\widetilde{X}^{\varepsilon, \omega}(t)=\varepsilon \widetilde{X}\left(\left\lfloor\varepsilon^{-2} t\right\rfloor\right)$. In order to prove (7.3), it suffices to show that, for $\eta, T>0$,

$$
P_{0}^{\omega}\left(\sup _{0 \leq t \leq T}\left|Y^{\varepsilon, \omega}(t)-\widetilde{X}^{\varepsilon, \omega}(t)\right|>\eta\right) \rightarrow 0 \quad \text { in } \mathbb{P}^{* *} \text {-probability as } \varepsilon \rightarrow 0
$$

and

$$
P_{0}^{\omega}\left(\sup _{0 \leq t \leq T}\left|\widetilde{X}^{\varepsilon, \omega}(t)-X^{\varepsilon, \omega}(t / m)\right|>\eta\right) \rightarrow 0 \quad \text { in } \mathbb{P}^{* *} \text {-probability as } \varepsilon \rightarrow 0 .
$$

Now,

$$
\begin{aligned}
\sup _{0 \leq t \leq T}\left|Y^{\varepsilon, \omega}(t)-\widetilde{X}^{\varepsilon, \omega}(t)\right| & \leq \max _{0 \leq s \leq \varepsilon^{-2} T}\left\{\varepsilon\left|Y^{\omega}(s)-\widetilde{X}^{\omega}(s)\right|\right\} \\
& \leq \varepsilon \max _{k: \theta_{k+1} \leq \varepsilon^{-2} T} \max _{\theta_{k} \leq s \leq \theta_{k+1}}\left|Y^{\omega}(s)-Y^{\omega}\left(\theta_{k}\right)\right| \\
& \leq \varepsilon \max _{k: \theta_{k+1} \leq \varepsilon^{-2} T} \mid \text { longest light path from } X^{\omega}(k) \text { to } X^{\omega}(k+1) \mid \\
& \leq \varepsilon \Lambda,
\end{aligned}
$$

where $\Lambda$ is the length of the longest light path intersecting the box $\left[-\varepsilon^{-2} T, \varepsilon^{-2} T\right]^{d}$. Therefore $P_{0}^{\omega}\left(\sup _{0 \leq t \leq T}\left|Y^{\varepsilon, \omega}(t)-\widetilde{X}^{\varepsilon, \omega}(t)\right|>\eta\right)=0$ unless $\Lambda>\eta / \varepsilon$. It follows that

$$
\mathbb{P}^{* *}\left[P_{0}^{\omega}\left(\sup _{0 \leq t \leq T}\left|Y^{\varepsilon, \omega}(t)-\widetilde{X}^{\varepsilon, \omega}(t)\right|>\eta\right)>\delta\right] \leq \mathbb{P}^{* *}\left(\Lambda>\frac{\eta}{\varepsilon}\right) \leq\left(2 T \varepsilon^{-2}\right)^{d} e^{-\xi \eta / \varepsilon},
$$

by Theorem 3.1, where $\xi>0$ is a constant. Equation (7.4) follows.

In proving (7.5), we use the following result, which follows by: standard manipulations using characterisation Lemma 7.1(c), the Arzelà-Ascoli characterisation of compact subsets of $C\left([0, \infty), \mathbb{R}^{d}\right)$, and the definition ([15], p. 117) of the Skorohod metric $\rho$ on the space $\Delta=D\left([0, \infty), \mathbb{R}^{d}\right)$. We omit the details of the proof.

Lemma 7.3. For given $T, \delta, \eta>0$, there exists $\gamma>0$ such that

$$
\mathbb{P}^{* *}\left[P_{0}^{\omega}\left(\sup _{0 \leq s, t \leq T,|s-t|<\gamma}\left|X^{\varepsilon, \omega}(t)-X^{\varepsilon, \omega}(s)\right|>\eta\right)>\delta\right] \rightarrow 0 \quad \text { as } \varepsilon \rightarrow 0 .
$$

If $0<\gamma<T$, we have that

$$
\begin{aligned}
\sup _{0 \leq t \leq T} \mid \widetilde{X}^{\varepsilon, \omega}(t)- & X^{\varepsilon, \omega}(t / m) \mid \\
& =\max \left\{\sup _{0 \leq t \leq \gamma}\left|\widetilde{X}^{\varepsilon, \omega}(t)-X^{\varepsilon, \omega}(t / m)\right|, \sup _{\gamma \leq t \leq T}\left|\widetilde{X}^{\varepsilon, \omega}(t)-X^{\varepsilon, \omega}(t / m)\right|\right\} .
\end{aligned}
$$

Note that $N(n) \leq n$, so that $\varepsilon^{2} N\left(\left\lfloor\varepsilon^{-2} t\right\rfloor\right) \leq t$ for $t \geq 0$. Since $m \geq 1, \widetilde{X}^{\varepsilon, \omega}(t)=$ $X^{\varepsilon, \omega}\left(\varepsilon^{2} N\left(\left\lfloor\varepsilon^{-2} t\right\rfloor\right)\right), X^{\varepsilon, \omega}(0)=0$, we have that

$$
\begin{aligned}
\sup _{0 \leq t \leq \gamma}\left|\widetilde{X}^{\varepsilon, \omega}(t)-X^{\varepsilon, \omega}(t / m)\right| & \leq \sup _{0 \leq t \leq \gamma}\left|X^{\varepsilon, \omega}(t)\right|+\sup _{0 \leq t \leq \gamma}\left|X^{\varepsilon, \omega}(t / m)\right| \\
& \leq 2 \sup _{0 \leq t \leq \gamma}\left|X^{\varepsilon, \omega}(t)\right| \leq 2 \sup _{\substack{0 \leq s, t \leq T \\
|s-t| \leq \gamma}}\left|X^{\varepsilon, \omega}(s)-X^{\varepsilon, \omega}(t)\right| .
\end{aligned}
$$


If $\left|\varepsilon^{2} N\left(\left\lfloor\varepsilon^{-2} t\right\rfloor\right)-t / m\right| \leq \gamma$ for $\gamma \leq t \leq T$, then

$$
\begin{aligned}
\sup _{\gamma \leq t \leq T}\left|\widetilde{X}^{\varepsilon, \omega}(t)-X^{\varepsilon, \omega}(t / m)\right| & =\sup _{\gamma \leq t \leq T}\left|X^{\varepsilon, \omega}\left(\varepsilon^{2} N\left(\left\lfloor\varepsilon^{-2} t\right\rfloor\right)\right)-X^{\varepsilon, \omega}(t / m)\right| \\
& \leq \sup _{\substack{0 \leq s, t \leq T \\
|s-t| \leq \gamma}}\left|X^{\varepsilon, \omega}(s)-X^{\varepsilon, \omega}(t)\right| .
\end{aligned}
$$

Therefore,

$$
\begin{aligned}
P_{0}^{\omega}\left(\sup _{0 \leq t \leq T}\left|\widetilde{X}^{\varepsilon, \omega}(t)-X^{\varepsilon, \omega}(t / m)\right|>\eta\right) \leq & P_{0}^{\omega}\left(\sup _{\substack{0 \leq s, t \leq T \\
|s-t| \leq \gamma}}\left|X^{\varepsilon, \omega}(s)-X^{\varepsilon, \omega}(t)\right|>\frac{1}{2} \eta\right) \\
& \left.+P_{0}^{\omega}\left(\sup _{\gamma \leq t \leq T} \mid m \varepsilon^{2} N\left(\mid \varepsilon^{-2} t\right\rfloor\right)-t \mid>m \gamma\right) .
\end{aligned}
$$

By Lemma 7.3, it suffices to show that, for $\gamma, \eta>0$ and $T>\gamma$,

$$
P_{0}^{\omega}\left(\sup _{\gamma \leq t \leq T}\left|m \varepsilon^{2} N\left(\left\lfloor\varepsilon^{-2} t\right\rfloor\right)-t\right|>\eta\right) \rightarrow 0 \quad \text { in } \mathbb{P}^{* *} \text {-probability as } \varepsilon \rightarrow 0 .
$$

This follows if we can show that, for $\eta>0$,

$$
P_{0}^{\omega}\left(\sup _{s \geq S_{0}}\left|\frac{m N(\lfloor s\rfloor)}{s}-1\right|>\eta\right) \rightarrow 0 \quad \text { in } \mathbb{P}^{* *} \text {-probability as } S_{0} \rightarrow \infty .
$$

Using standard arguments from renewal theory, it suffices to prove that, for $\delta>0$,

$$
P_{0}^{\omega}\left(\sup _{k \geq K_{0}}\left|\frac{\theta_{k}}{k}-m\right|>\delta\right) \rightarrow 0 \quad \text { in } \mathbb{P}^{* *} \text {-probability as } K_{0} \rightarrow \infty \text {. }
$$

It remains to establish (7.6). Let

$$
f_{K}=\sup _{k \geq K}\left|\frac{\theta_{k}}{k}-m\right|
$$

Since a.s. convergence implies convergence in probability, it suffices to show that, for $\mathbb{P}^{* *}$-a.e. $\omega$, we have that $f_{K} \rightarrow 0 P_{0}^{\omega}$-a.s.; the rest of the proof is devoted to proving this fact.

Suppose $\xi_{0} \in \Omega^{* *}=\left\{\omega \in \Omega: 0 \in \mathcal{W}(\omega),\left|C_{0}(\omega)\right|=\infty\right\}$ is distributed according to $\mathbb{P}^{* *}$, and suppose that $\eta_{0}, \eta_{1}, \ldots$ is a sequence of random variables independent of $\xi_{0}$ and of each other, each of which is uniformly distributed on the set $I^{ \pm}=\left\{ \pm u_{i}: i=1,2, \ldots, d\right\}$. For $n \geq 0$, let $\xi_{n+1}=\tau_{-x} \xi_{n}$ where $x \in \mathbb{Z}^{d}$ is the first rw point encountered when proceeding along the light path starting at 0 in the direction $\eta_{n}$, within the environment $\xi_{n}$. [The shift $\tau_{-x}$ was defined above Lemma 5.2.]

Let $\zeta_{n}=\left(\xi_{n}, \eta_{n}\right)$. Then $\left(\zeta_{n}: n \geq 0\right)$ is a stationary Markov chain on $\aleph^{* *}=\Omega^{* *} \times I^{ \pm}$ having invariant measure $\mathbb{P}^{* *} \times \nu$, where $\nu$ is the uniform measure on $I^{ \pm}$. Let $\widetilde{A} \subseteq \aleph^{* *}$ be invariant under the action of the chain $\left(\zeta_{n}\right)$. It may be shown that $\widetilde{A}$ has the form $A \times I^{ \pm}$ for some $A\left(\subseteq \Omega^{* *}\right)$ which is invariant under the action of the chain $\left(\xi_{n}\right)$. It follows from the fact that the chain $\left(\xi_{n}\right)$ is ergodic that $\left(\zeta_{n}\right)$ is an ergodic chain also. Following the argument of [12] (Thm 1.1, and the preceding remarks on pp. 459-460) we deduce that $\left(\zeta_{n}\right)$ is an ergodic stationary sequence. 
Let $\lambda_{n}=l\left(\zeta_{n}\right)$ where $l: \aleph^{* *} \rightarrow \mathbb{N}$ was defined at the beginning of this section. Since $\left(\zeta_{n}\right)$ is an ergodic stationary sequence, the same is true of $\left(\lambda_{n}\right)$. Therefore,

$$
\frac{1}{n} \theta_{n}=\frac{1}{n} \sum_{k=0}^{n} \lambda_{k} \rightarrow m \quad \text { a.s. }
$$

by (7.1) and the ergodic theorem. Using Fubini's theorem, the 'a.s. a.s.' version of (7.6) follows.

Acknowledgements. This work was aided by partial financial support from the European Union under contracts CHRX-CT93-0411 and FMRX-CT96-0075A, and from the Engineering and Physical Sciences Research Council under grant GR/L15426. It was done during a visit by C.E.B. to the University of Cambridge while on sabbatical leave from the University of Rochester.

\section{REFERENCES}

1. Antal, P. and Pisztora, A., On the chemical distance for supercritical Bernoulli percolation, Annals of Probability 24 (1996), 1036-1048.

2. Beijeren, H. van, Transport properties of stochastic Lorentz models, Reviews in Modern Physics 54 (1982), 195-234.

3. Beijeren, H. van and Spohn, H., Transport properties of the one dimensional stochastic Lorentz model. I. Velocity autocorrelation, Journal of Statistical Physics 31 (1982), 231-254.

4. Billingsley, P., Convergence of Probability Measures, Wiley, New York, 1968.

5. Bunimovich, L. A. and Troubetzkoy, S. E., Recurrence properties of Lorentz lattice gas cellular automata, Journal of Statistical Physics 67 (1992), 289-302.

6. Burton, R. M. and Keane, M., Density and uniqueness in percolation, Communications in Mathematical Physics 121 (1989), 501-505.

7. Cohen, E. G. D., New types of diffusions in lattice gas cellular automata, Microscopic Simulations of Complex Hydrodynamical Phenomena (M. Mareschal and B. L. Holian, eds.), Plenum Press, New York, 1991, pp. 137-152.

8. Cohen, E. G. D. and Wang, F., New results for diffusion in Lorentz lattice gas cellular automata, Journal of Statistical Physics 81 (1995), 445-466.

9. Cohen, E. G. D. and Wang, F., Novel phenomena in Lorentz lattice gases, Physica A 219 (1995), 56-87.

10. DeMasi, A., Ferrari, P. A., Goldstein, S., and Wick, W. D., Invariance principle for reversible Markov processes with application to diffusion in the percolation regime, Particle Systems, Random Media and Large Deviations (R. T. Durrett, ed.), Contemporary Mathematics no. 41, American Mathematical Society, Providence, R. I., 1985, pp. 71-85.

11. DeMasi, A., Ferrari, P. A., Goldstein, S., and Wick, W. D., An invariance principle for reversible Markov processes. Applications to random motions in random environments, Journal of Statistical Physics $\mathbf{5 5}$ (1989), 787-855.

12. Doob, J. L., Stochastic Processes, Wiley, New York, 1953.

13. Doyle, P. G. and Snell, E. L., Random Walks and Electric Networks, Carus Mathematical Monograph no. 22, AMA, Washington, D. C, 1984.

14. Ehrenfest, P., Collected Scientific Papers (M. J. Klein, ed.), North-Holland, Amsterdam, 1959.

15. Ethier, S. N. and Kurtz, T. G., Markov Processes, Characterization and Convergence, Wiley, New York, 1986.

16. Goldstein, S., Antisymmetric functionals of reversible Markov processes, Annales de l'Institut Henri Poincaré, Probabilités et Statistiques 31 (1995), 177-190.

17. Grimmett, G. R., Percolation, Springer-Verlag, Berlin, 1989.

18. Grimmett, G. R., Percolation and disordered systems, Ecole d'Eté de Probabilités de Saint Flour XXVI1996 (P. Bernard, ed.), Lecture Notes in Mathematics no. 1665, Springer, Berlin, 1997, pp. 153-300.

19. Grimmett, G. R. and Kesten, H., First-passage percolation, network flows and electrical networks, Zeitschrift für Wahrscheinlichkeitstheorie und Verwandte Gebiete 66 (1984), 335-366.

20. Grimmett, G. R. and Marstrand, J. M., The supercritical phase of percolation is well behaved, Proceedings of the Royal Society (London), Series A 430 (1990), 439-457.

21. Grimmett, G. R., Menshikov, M. V., and Volkov, S. E., Random walks in random labyrinths, Markov Processes and Related Fields 2 (1996), 69-86. 
22. Hollander, F. den, Naudts, J., and Redig, F., Invariance principle for the stochastic Lorentz lattice gas, Journal of Statistical Physics 66 (1992), 1583-1598.

23. Kesten, H., Percolation Theory for Mathematicians, Birkhäuser, Boston, 1982.

24. Kipnis, C. and Varadhan, S. R. S., Central limit theorem for additive functionals of reversible Markov processes and applications to simple exclusion, Communications in Mathematical Physics 104 (1986), $1-19$.

25. Liggett, T. M., Schonmann, R. H., and Stacey, A., Domination by product measures, Annals of Probability 25 (1997), 71-95.

26. Lorentz, H. A., The motion of electrons in metallic bodies, I, II, and III, Koninklijke Akademie van Wetenschappen te Amsterdam, Section of Sciences 7 (1905), 438-453, 585-593, 684-691.

27. Pisztora, A., Surface order large deviations for Ising, Potts and percolation models, Probability Theory and Related Fields 104 (1996), 427-466.

28. Quas, A., Infinite paths in a Lorentz lattice gas model (1996) (to appear).

29. Tóth, B., Persistent random walks in random environment, Probability Theory and Related Fields $\mathbf{7 1}$ (1986), 615-625.

30. Wang, F. and Cohen, E. G. D., Diffusion in Lorentz lattice gas cellular automata: the honeycomb and quasi-lattices compared with the square and triangular lattices, Journal of Statistical Physics 81 (1995), 467-495.

31. Ziff, R. M., Kong, X. P., and Cohen, E. G. D., Lorentz lattice-gas and kinetic-walk model, Physical Review A 44 (1991), 2410-2428.

Carol Bezuidenhout, Centre de Mathematiques et Informatique, Technopole de Chateau Gombert, 39, Rue Joliot Curie, 13453 Marseile Cedex 13, France

E-mail address: cbez@gyptis.univ-mrs.fr

Geoffrey Grimmett, Statistical Laboratory, University of Cambridge, 16 Mill Lane, CamBRIDGE CB2 1SB, UK

E-mail address: g.r.grimmett@statslab.cam.ac.uk

$U R L:$ http://www.statslab.cam.ac.uk/ grg/ 